\title{
Le impugnazioni cautelari nel recente "attivismo" delle Sezioni unite della Corte di cassazione
}

\author{
Appeals against precautionary measures in the recent \\ "activism" of the Joint Chambers of the Court of Cassation
}

\section{Marta Bargis ${ }^{1}$}

Università del Piemonte Orientale, Alessandria, Italia

marta.bargis@uniupo.it

https://orcid.org/0000-0002-9913-7124

\begin{abstract}
AвSTRACt: Il saggio si occupa delle impugnazioni cautelari esaminandole da uno specifico angolo visuale, quello del bilanciamento fra le esigenze di celerità e la tutela del diritto di difesa dell'indagato e dell'imputato. A questo fine, vengono analizzate tre recenti decisioni delle Sezioni unite della Corte di cassazione, che documentano posizioni oscillanti della Suprema Corte e richiederebbero interpretazioni autentiche da parte del legislatore.

Parole chiave: impugnazioni cautelari; esigenze di celerità; diritto di difesa dell'indagato e dell'imputato; assenza della vittima; recenti pronunce delle Sezioni unite della Corte di cassazione.

ABSTRACT: The paper examines appeals against precautionary measures from the specific standpoint of the balance between promptness and right to defence for suspect and accused. For this purpose, three recent judgments of the Joint Chambers of the Court of Cassation are analyzed: the oscillating solutions adopted by the Supreme Court would require an authentic legislative interpretation.

KEYWORDs: appeals against precautionary measures; promptness; right to defence for suspect and accused; victim's absence; recent judgments of the Joint Chambers of the Court of Cassation.
\end{abstract}

Sommario: Premessa; 1. Lo stato dell'arte: le impugnazioni cautelari al bivio tra esigenze di celerità e tutela del diritto di difesa dell'imputato.

1 Professore emerito di diritto processuale penale - Università degli Studi del Piemonte Orientale. 
L'assenza della vittima; 2 . Gli ultimi nodi sciolti dalle Sezioni unite della Corte di cassazione: riesame e comparizione personale dell'indagato/imputato (art. 309 commi 6 e 8-bis c.p.p.); 3. Segue: misura cautelare coercitiva disposta su appello del pubblico ministero (art. 310 commi 1 e 3 c.p.p.) e interrogatorio di garanzia; 4. Segue: giudizio di riesame in sede di rinvio e decorrenza del termine di dieci giorni dalla «ricezione degli atti» per la decisione (art. 311 comma 5-bis c.p.p.); Conclusioni; Bibliografia.

\section{Premessa}

Le impugnazioni cautelari costituiscono un importante banco di prova nel sistema delle misure restrittive della libertà individuale, come è dimostrato dall'attenzione loro riservata dalla 1.16 aprile 2015, n. 47, che, nell'introdurre rilevanti modifiche alla disciplina del microcosmo cautelare, è specificamente intervenuta in tema di riesame (art. 309 c.p.p.), di appello (art. 310 c.p.p.) e di ricorso per cassazione (art. 311 c.p.p.) ${ }^{2}$.

L'applicazione pratica della riforma del 2015, dall'angolo visuale qui prescelto, non è andata esente da criticità interpretative. Nel presente saggio se ne esamineranno alcune, che si sono tradotte in altrettante questioni sulle quali le Sezioni unite della Corte di cassazione sono state chiamate a pronunciarsi nel corso del $2020^{3}$; ad esse vanno affiancate altre questioni non dipendenti dalla riforma de qua e, pertanto, non sottoposte ad analisi in questa sede 4 . A un globale sguardo d'insieme, si è così concretizzato un notevole "attivismo" del Supremo consesso.

2 Per un quadro generale v. MARZADURI, Enrico. Diritto di difesa e tempi del procedimento dinanzi al tribunale della libertà. In: GIULIANI, Livia (a cura di). La riforma delle misure cautelari personali. Torino: Giappichelli, 2015, p. 211 ss. e, più di recente, MAGGIO, Paola. Le impugnazioni delle misure cautelari personali. Milano: Giuffrè Francis Lefebvre, 2018, passim.

3 Si tratta delle questioni illustrate infra, §§ 2, 3 e 4 .

4 Il riferimento va a due pronunce delle Sezioni unite. La prima è Cass., Sez. un., 23 aprile 2020, n. 19214, p.m. in c. Giacobbe, che ha affermato il principio di diritto secondo cui sussiste «l'interesse del pubblico ministero ad impugnare il provvedimento con il quale il tribunale del riesame, rilevata l'incompetenza 
Prima di vagliare i profili affrontati dalle Sezioni unite concernenti, da varie prospettive, tutti i rimedi cautelari - non si può che muovere dall'odierno stato dell'arte di tali rimedi ${ }^{5}$ : il fil rouge che li percorre sembra tracciare un percorso ancipite, nel difficile bilanciamento fra le esigenze di celerità che connotano il procedimento di impugnazione cautelare e la tutela del diritto di difesa dell'indagato/imputato.

Solo dopo avere analizzato le pronunce delle Sezioni unite, sarà possibile, in sede conclusiva, verificare come un simile fil rouge sia stato dipanato dalla Suprema Corte nella sua massima composizione.

\section{LO STATO DELL'ARTE: LE IMPUGNAZIONI CAUTELARI AL BIVIO TRA ESIGENZE DI CELERITÀ E TUTELA DEL DIRITTO DI DIFESA DELL'IMPUTATO. L'ASSENZA DELLA VITTIMA}

Come detto nella Premessa, la riforma del 2015 ha interessato anche la tematica delle impugnazioni cautelari: numerose e incisive le

del giudice per le indagini preliminari, annulli, per carenza delle condizioni di applicabilità, l'ordinanza con cui quello stesso giudice ha disposto la misura cautelare della custodia in carcere, se l'impugnazione è funzionale a garantire il tempestivo intervento del giudice competente» (per un commento v. ALVINO, Francesco. Incompetenza per territorio, urgenza cautelare e interesse del pubblico ministero alle relative contestazioni nelle impugnazioni de libertate. Sistema penale, n. 9, p. 125 ss., 2020; BLEFARI, Chiara Rosa. Le Sezioni Unite si pronunciano sull'interesse a ricorrere del Pubblico ministero, nel caso in cui il Giudice del riesame, rilevata l'incompetenza del Giudice che ha emesso l'ordinanza, la annulli. Processo penale e giustizia, p. 106 ss., 2021). La seconda è Cass., Sez. un., 24 settembre 2020, n. 1626, Bottari, a parere della quale «il ricorso cautelare per cassazione avverso la decisione del tribunale del riesame $o$, in caso di ricorso immediato, del giudice che ha emesso la misura, deve essere presentato esclusivamente presso la cancelleria del tribunale che ha emesso la decisione o, nel caso indicato dall'art. 311 comma 2 c.p.p., del giudice che ha emesso l'ordinanza, ponendosi a carico del ricorrente il rischio che l'impugnazione, presentata ad un ufficio diverso da quello indicato dalla legge, sia dichiarata inammissibile per tardività, in quanto la data di presentazione rilevante ai fini della tempestività è quella in cui l'atto perviene all'ufficio competente a riceverlo» (per un commento v. DI DOMENICO, Alessia. Le Sezioni Unite sul deposito del ricorso in sede cautelare: un'occasione per chiarire la corretta interpretazione dell'art. 311 c.p.p. Sistema penale, 4 febbraio 2021; GERARDI Alessandro. Il luogo delle impugnazioni cautelari di legittimità: la stretta delle Sezioni unite. Archivio penale [web], n. 1, p. 1 ss., 2021).

5 Cfr. infra, § 1. 
incursioni nella disciplina del riesame (art. 309 c.p.p. $)^{6}$, più circoscritte e attenuate quelle incidenti sull'appello cautelare (art. 310 c.p.p.) ${ }^{7}$, comunque di rilievo la novità che attiene al giudizio di rinvio dopo l'annullamento da parte della Corte di cassazione (art. 311 c.p.p.) ${ }^{8}$.

In linea generale, il problema di fondo è costituito, oggi come in passato, dal rapporto fra «gli spazi assicurati alla difesa e la tempestività del sindacato sull'ordinanza cautelare» ${ }^{9}$. Cominciando dal riesame, la prima innovazione concerne il riconoscimento del diritto dell'imputato a partecipare personalmente all'udienza di riesame (art. 309 commi 6 primo periodo e 8-bis secondo periodo c.p.p. $)^{10}$ ed è stata sottoposta allo scrutinio delle Sezioni unite della Corte di cassazione ${ }^{11}$. L'altra novità, per certi versi collegata alla precedente (le stesse Sezioni unite ne valorizzano il legame), è contenuta nel nuovo comma 9-bis dell'art. 309 c.p.p., che

6 L'art. 11 1. n. 47 del 2015 ha infatti operato nell'art. 309 c.p.p. interpolazioni (commi 6, 8-bis e 9), inserimenti (nuovo comma 9-bis) e sostituzioni (comma 10).

7 L'art. 12 1. n. 47 del 2015 si è limitato a interpolare il comma 2 dell'art. 310 c.p.p., trasponendo - con variazioni - quanto stabilito per il riesame nell'art. 309 comma 10 secondo e terzo periodo c.p.p.

L'art. 13 1. n. 47 del 2015 ha aggiunto nell'art. 311 c.p.p. il nuovo comma 5-bis.

9 Così MARZADURI, Enrico. Diritto di difesa e tempi del procedimento dinanzi al tribunale della libertà. In: GIULIANI, Livia (a cura di). La riforma delle misure cautelari personali. Torino: Giappichelli, 2015, p. 211. Sulle pronunce della Corte europea dei diritti dell'uomo che hanno ritenuto necessaria la tempestività della decisione in sede di rimedi preordinati al controllo sulla legalità della detenzione cautelare v. MAGGIO, Paola. Le impugnazioni delle misure cautelari personali. Milano: Giuffrè Francis Lefebvre, 2018, p. 59-62; RAFARACI, Tommaso. Le garanzie del procedimento de libertate: il diritto ad ottenere in un termine congruo le decisioni sulla libertà personale. In: BALSAMO, Antonio; KOSTORIS, Roberto E. (a cura di). Giurisprudenza europea e processo penale italiano. Torino: Giappichelli, 2008, p. 271 ss. Per un quadro d'insieme delle garanzie minime elaborate dalla giurisprudenza di Strasburgo a tutela della libertà personale dell'imputato v. altresì GIULIANI, Livia. Autodifesa e difesa tecnica nei procedimenti de libertate. Padova: Cedam, 2012, p. 190-198.

10 Su questo aspetto v. MARZADURI, Enrico. Diritto di difesa e tempi del procedimento dinanzi al tribunale della libertà. In: GIULIANI, Livia (a cura di). La riforma delle misure cautelari personali. Torino: Giappichelli, 2015, p. 217224; nonché MAGGIO, Paola. Le impugnazioni delle misure cautelari personali. Milano: Giuffrè Francis Lefebvre, 2018, p. 209-212.

11 V. infra, § 2. 
consente all'imputato di formulare personalmente, entro due giorni dalla notificazione dell'avviso, la richiesta di differimento ${ }^{12}$ della data dell'udienza, da un minimo di cinque a un massimo di dieci giorni, «se vi siano giustificati motivi» ${ }^{13}$ : l'accoglimento della richiesta comporta che il termine per la decisione e quello per il deposito dell'ordinanza siano prorogati in eguale misura. Pare evidente che il legislatore abbia in tal modo perseguito un bilanciamento fra la celerità che dovrebbe caratterizzare l'impugnazione cautelare e l'effettiva realizzazione del diritto di difesa, riconoscendo all'imputato la facoltà di chiedere una diversa tempistica del procedimento di riesame.

Infine, nel testo del comma 10 dell'art. 309 c.p.p. si è previsto un altro termine perentorio inerente al deposito dell'ordinanza del tribunale della libertà («trenta giorni dalla decisione salvi i casi in cui la stesura della motivazione sia particolarmente complessa per il numero degli arrestati o la gravità delle imputazioni», nei quali il giudice «può disporre per il deposito un termine più lungo, comunque non eccedente il quarantacinquesimo giorno da quello della decisione») ${ }^{14}$. Nel medesimo

12 Sull'istituto v. ILLUMINATI, Giulio. Verso il ripristino della cultura delle garanzie in tema di libertà personale dell'imputato. Rivista italiana di diritto $e$ procedura penale, p. 1157 s., 2015; MARZADURI, Enrico. Diritto di difesa e tempi del procedimento dinanzi al tribunale della libertà. In: GIULIANI, Livia (a cura di). La riforma delle misure cautelari personali. Torino: Giappichelli, 2015, p. 226-230; nonché MAGGIO, Paola. Le impugnazioni delle misure cautelari personali. Milano: Giuffrè Francis Lefebvre, 2018, p. 229-231.

13 Silente la disposizione sull'ambito di tali «giustificati motivi», si è suggerito che le ragioni del differimento «debbano essere rinvenute principalmente nella necessità di una tempistica meno serrata per procedere agli opportuni approfondimenti nello studio e nell'analisi del materiale probatorio»: MARZADURI, Enrico. Diritto di difesa e tempi del procedimento dinanzi al tribunale della libertà. In: GIULIANI, Livia (a cura di). La riforma delle misure cautelari personali. Torino: Giappichelli, 2015, p. 229; cfr. ILLUMINATI, Giulio. Verso il ripristino della cultura delle garanzie in tema di libertà personale dell'imputato. Rivista italiana di diritto e procedura penale, p. 1157, 2015. Sul punto, per alcune esemplificazioni, v. SPANGHER, Giorgio. Un restyling per le misure cautelari. Diritto penale e processo, p. 532, 2015. V. anche infra, § 2 e nota 51.

${ }_{14}$ In argomento v. MARZADURI, Enrico. Diritto di difesa e tempi del procedimento dinanzi al tribunale della libertà. In: GIULIANI, Livia (a cura di). La riforma delle misure cautelari personali. Torino: Giappichelli, 2015, p. 231-234; nonché MAGGIO, Paola. Le impugnazioni delle misure cautelari personali. Milano: Giuffrè Francis Lefebvre, 2018, p. 356 s. 
comma 10 dell'art. 309 c.p.p. è poi stata regolamentata in modo rigido l'evenienza di una rinnovazione del provvedimento nei casi di perdita di efficacia dell'ordinanza che ha disposto la misura coercitiva per inosservanza dei termini prescritti per la trasmissione degli atti ex art. 309 comma 5 c.p.p., per la decisione sulla richiesta di riesame e per il deposito dell'ordinanza in cancelleria ${ }^{15}$.

Passando all'appello cautelare, la riforma del 2015 ha introdotto nell'art. 310 comma 2 c.p.p. la previsione secondo cui l'ordinanza va depositata in cancelleria entro trenta giorni dalla decisione, salvi i casi in cui «la stesura della motivazione sia particolarmente complessa per il numero degli arrestati o la gravità delle imputazioni», nei quali «il giudice può indicare nel dispositivo un termine più lungo, non eccedente comunque il quarantacinquesimo giorno da quello della decisione» ${ }^{16}$. L'apparentamento con l'analoga previsione relativa al riesame è solo parziale $^{17}$ : quanto all'appello, infatti, i termini per il deposito dell'ordinanza non sono perentori ma ordinatori ${ }^{18}$, in armonia, del resto, con il carattere ordinatorio del termine di venti giorni dalla ricezione degli atti entro il quale il tribunale della libertà decide in veste di giudice di appello.

Va inoltre sin d'ora osservato che, non essendo richiamati dall'art. 310 comma 2 primo periodo c.p.p. i commi 6 primo periodo,

15 Su questo profilo v. MARZADURI, Enrico. Diritto di difesa e tempi del procedimento dinanzi al tribunale della libertà. In: GIULIANI, Livia (a cura di). La riforma delle misure cautelari personali. Torino: Giappichelli, 2015, p. 235 ss.

16 Per un commento v. BARGIS, Marta. L'appello cautelare: un innesto per rifrazione. Disponibile in: http://www.lalegislazionepenale.eu, 22 settembre 2015.

17 Circa talune disarmonie v. BARGIS, Marta. L'appello cautelare: un innesto per rifrazione. Disponibile in: http://www.lalegislazionepenale.eu, 22 settembre 2015, p. 3, nota 12.

18 Cfr. BARGIS, Marta. L'appello cautelare: un innesto per rifrazione. Disponibile in: http://www.lalegislazionepenale.eu, 22 settembre 2015, p. 4-6; ILLUMINATI, Giulio. Verso il ripristino della cultura delle garanzie in tema di libertà personale dell'imputato. Rivista italiana di diritto e procedura penale, $\mathrm{p}$. 1160, 2015; MAGGIO, Paola. Le impugnazioni delle misure cautelari personali. Milano: Giuffrè Francis Lefebvre, 2018, p. 246; MARZADURI, Enrico. Diritto di difesa e tempi del procedimento dinanzi al tribunale della libertà. In: GIULIANI, Livia (a cura di). La riforma delle misure cautelari personali. Torino: Giappichelli, 2015, p. 232 e nota 81; SPANGHER, Giorgio. Un restyling per le misure cautelari. Diritto penale e processo, p. 534, 2015. 
8-bis secondo periodo e 9-bis dell'art. 309 c.p.p., l'imputato non ha il diritto di comparire personalmente all'udienza (varranno comunque le forme previste dall'art. 127 c.p.p., come stabilisce l'art. 310 comma 2 terzo periodo c.p.p. $)^{19}$ o di chiederne il differimento. Altresì con riguardo all'appello cautelare si sono pronunciate le Sezioni unite ${ }^{20}$ : sebbene il profilo venuto in evidenza non sia stato toccato dalla riforma del 2015, vedremo che a tale riforma il Supremo consesso si è riferito, sia in generale, sia reputando, discutibilmente, che l'imputato abbia il diritto di comparire di persona all'udienza dell'appello cautelare.

Di precipuo risalto è infine l'intervento operato dalla l. n. 47 del 2015 sull'art. 311 c.p.p.: il nuovo comma 5-bis, che concerne il giudizio di rinvio dopo l'annullamento da parte della Corte di cassazione ${ }^{21}$, su ricorso dell'imputato, di un'ordinanza che ha disposto o confermato la misura coercitiva ai sensi dell'art. 309 comma 9 c.p.p., statuisce che il giudice di rinvio «decide entro dieci giorni dalla ricezione degli atti e l'ordinanza è depositata in cancelleria entro trenta giorni dalla decisione»; se la decisione o il deposito non intervengono nei termini prescritti, «l'ordinanza che ha disposto la misura coercitiva perde efficacia, salvo che l'esecuzione sia sospesa ai sensi dell'articolo 310, comma 3, e, salve eccezionali esigenze cautelari specificamente motivate, non può essere rinnovata». Non c'è dubbio che con l'innovato testo dell'art. 309 comma 10 c.p.p. e con l'inserimento del nuovo comma 5-bis nell'art. 311 c.p.p. (al primo ispirato), la 1. n. 47 del 2015 «abbia inteso affrontare in maniera unitaria la tematica delle impugnazioni cautelari, in modo da rendere più certa la

19 In tal senso MARZADURI, Enrico. Diritto di difesa e tempi del procedimento dinanzi al tribunale della libertà. In: GIULIANI, Livia (a cura di). La riforma delle misure cautelari personali. Torino: Giappichelli, 2015, p. 224 s. e nota 52. Per considerazioni critiche v. MAGGIO, Paola. Le impugnazioni delle misure cautelari personali. Milano: Giuffrè Francis Lefebvre, 2018, p. 247 s.

$20 \mathrm{~V}$. infra, §3.

21 Per un commento v. BARGIS, Marta. Le novità nella disciplina del giudizio di rinvio: una replica imperfetta. Disponibile in: http://www.lalegislazionepenale.eu, 22 settembre 2015. V. altresì MAGGIO, Paola. Le impugnazioni delle misure cautelari personali. Milano: Giuffrè Francis Lefebvre, 2018, p. 495 ss.; MARZADURI, Enrico. Diritto di difesa e tempi del procedimento dinanzi al tribunale della libertà. In: GIULIANI, Livia (a cura di). La riforma delle misure cautelari personali. Torino: Giappichelli, 2015, p. 243 ss. 
tempistica del giudizio di riesame (anche in sede di rinvio) ed effettiva la previsione della perdita di efficacia conseguente all'inosservanza dei termini perentori fissati» ${ }^{22}$.

Pure il comma 5-bis dell'art. 311 c.p.p. è approdato sotto la lente delle Sezioni unite ${ }^{23}$, al fine di interpretare la locuzione «ricezione degli atti», momento dal quale si computa il termine perentorio di dieci giorni per la decisione del giudice di rinvio.

Per ragioni di completezza, prima di concludere questa breve carrellata sullo stato dell'arte delle impugnazioni cautelari, va ricordato che sul proscenio non compare la vittima del reato. Ł̀ una esclusione che desta perplessità, se posta a confronto con la disciplina della revoca e della sostituzione delle misure cautelari, dove si configura invece un coinvolgimento della persona offesa e del suo difensore (art. 299 commi 2-bis, 3 secondo, terzo e quarto periodo e 4-bis secondo periodo c.p.p.), con riguardo alle misure coercitive previste dagli artt. 282-bis, 282-ter, 283, 284, 285 e 286 c.p.p., applicate nei procedimenti aventi ad oggetto delitti commessi con violenza alla persona. In tal caso, il difensore e la persona offesa possono, nei due giorni successivi alla notifica della richiesta di revoca o di sostituzione delle predette misure coercitive, «presentare memorie» ai sensi dell'art. 121 c.p.p. (art. 299 comma 3 terzo periodo c.p.p.) ${ }^{24}$ : condivisibile l'opinione,

22 In questi termini BARGIS, Marta. Le novità nella disciplina del giudizio di rinvio: una replica imperfetta. Disponibile in: http://www.lalegislazionepenale. eu, 22 settembre 2015, p. 11. Opinione ripresa da Corte cost., 21 settembre 2016-3 novembre 2016, n. 233 (punto 4 del Considerato in diritto), che ha dichiarato non fondata la questione di legittimità costituzionale dell'art. 309 comma 10 c.p.p., sollevata, in riferimento agli artt. 3, 101 comma 2 e 104 comma 1 Cost., «nella parte in cui prevede che l'ordinanza che dispone una misura coercitiva - diversa dalla custodia in carcere - che abbia perso efficacia non possa essere reiterata salve eccezionali esigenze cautelari specificamente motivate»: per un commento v. ANGIOLINI, Giulia. Il "nuovo" art. 309 comma 10 c.p.p. supera l'esame di costituzionalità (ma non ancora quello della prassi applicativa). Diritto penale contemporaneo, 6 dicembre 2016. V. infra, § 4.

Rilievi critici sulla disciplina, in relazione tanto alla persona offesa quanto all'imputato, sono espressi da ZACCHÈ, Francesco. Le cautele fra prerogative dell'imputato e tutela della vittima di reati violenti. Rivista italiana di diritto e procedura penale, p. 675-677, 2015. 
espressa in dottrina, che, se si consente di «incidere sull'esito di una richiesta di revoca con la presentazione di memorie», sembrerebbe ragionevole prevedere «facoltà analoghe» allorché l'imputato «cerca di raggiungere il medesimo risultato liberatorio con la proposizione dell'istanza di riesame ${ }^{25}$.

\section{Gli ultimi nodi sciolti dalle Sezioni unite della Corte di CASSAZIONE: RIESAME E COMPARIZIONE PERSONALE DELL'INDAGATO/ IMPUTATO (ART. 309 COMMI 6 E 8-BIS C.P.P.)}

Come già sappiamo ${ }^{26}$, la riforma del 2015 ha previsto per l'imputato ${ }^{27}$ la possibilità di chiedere la comparizione personale in udienza: infatti, l'art. 309 comma 6 primo periodo c.p.p. stabilisce che «con la richiesta di riesame» l'imputato «può chiedere di comparire personalmente», mentre il comma 8-bis secondo periodo del medesimo articolo dispone che l'imputato il quale «ne abbia fatto richiesta ai sensi del comma 6 ha diritto di comparire personalmente». Il combinato disposto ha dato origine in giurisprudenza a due orientamenti interpretativi ${ }^{28}$, che hanno reso necessario l'intervento delle Sezioni unite.

Secondo l'orientamento di gran lunga maggioritario, che risale al periodo immediatamente successivo all'entrata in vigore della $1 . \mathrm{n}$.

25 In tale direzione v. MARZADURI, Enrico. Diritto di difesa e tempi del procedimento dinanzi al tribunale della libertà. In: GIULIANI, Livia (a cura di). La riforma delle misure cautelari personali. Torino: Giappichelli, 2015, p. 225. Adesivamente cfr. MAGGIO, Paola. Le impugnazioni delle misure cautelari personali. Milano: Giuffrè Francis Lefebvre, 2018, p. 212 s.; PAULESU, Pier Paolo. Vittima del reato e processo penale: uno sguardo d'insieme (informazioni, diritti, tutele). In: BARGIS, Marta; BELLUTA, Hervé (a cura di), Vittime di reato e sistema penale. Torino: Giappichelli, 2017, p. 158. Cfr. supra, § 1 .

La previsione si estende alla persona sottoposta alle indagini (art. 61 comma 1 c.p.p.).

28 Descritti nell'ordinanza di rimessione, Cass., sez. V, ord. 13 settembre 2019, n. 43406, Ramondo. Sistema penale, 5 febbraio 2020. A commento v. GATTO, Antonio. Il diritto dell'imputato detenuto di presenziare all'udienza di riesame al vaglio delle Sezioni unite. Sistema penale, n. 2, p. 17 ss., 2020. 
47 del 2015 ma è stato ribadito in tempi più recenti $\mathrm{i}^{29}$, la richiesta in discorso va formulata esclusivamente con l'istanza di riesame (anche dal difensore, se quest'ultima è da lui presentata), mentre non sono più applicabili le disposizioni degli artt. 127 comma 3 c.p.p. e 101 disp. att. c.p.p., che prevedono il diritto dell'interessato detenuto o internato fuori dal circondario a essere sentito dal magistrato di sorveglianza. In altri termini, il novum normativo ha introdotto, senza alcuna distinzione, il diritto dell'interessato di comparire all'udienza camerale, ancorandone tuttavia l'esercizio «ad un dato obiettivo ed incontrovertibile», in modo da rispettare $i$ «ritmi snelli e serrati» del procedimento di riesame, sottrarre alla discrezionalità del giudice «l'apprezzamento della "tempestività" della richiesta di comparizione» e scoraggiare «atteggiamenti dilatori od ostruzionistici da parte del ristretto». D'altro canto, se l'espressione della volontà di partecipare all'udienza non fosse soggetta a limiti temporali, l'effettiva tutela di questo diritto fondamentale finirebbe con il dipendere «dalla capacità organizzativa dell'ufficio giudiziario», con le inevitabili disomogeneità sul piano territoriale. Viceversa, l'avere agganciato la richiesta alla presentazione di quella di riesame realizza un «equilibrato bilanciamento tra la tutela del diritto di partecipazione e l'istanza acceleratoria che connota il procedimento di riesame», nel rispetto del principio di eguaglianza.

Negli anni più vicini, peraltro, alcune pronunce hanno espresso un diverso parere ${ }^{30}$. Muovendo dalla premessa che la riforma del 2015 ha inteso rafforzare il diritto dell'interessato a comparire nell'udienza del procedimento di riesame, rispetto a un sistema che lo riconosceva al

29 Per i necessari richiami (ai quali adde Cass., sez. V, 26 giugno 2019, n. 34181, Basso) v. Cass., sez. V, ord. 13 settembre 2019, n. 43406, Ramondo. Sistema penale, 5 febbraio 2020, punto 3 del Considerato in diritto, cui si riferiscono le citazioni nel testo. Quanto all'analisi di tale orientamento e alla indicazione di ulteriori sentenze v. la decisione delle Sezioni unite citata infra, nota 31. Numerose segnalazioni, relative a pronunce non massimate, sono fornite da GATTO, Antonio. Il diritto dell'imputato detenuto di presenziare all'udienza di riesame al vaglio delle Sezioni unite. Sistema penale, n. 2, p. 21, nota 13, 2020.

30 L'orientamento appare peraltro minoritario: per gli opportuni richiami v. Cass., sez. V, ord. 13 settembre 2019, n. 43406, Ramondo. Sistema penale, 5 febbraio 2020, punto 2 del Considerato in diritto, al quale si collegano le citazioni nel testo. 
solo soggetto detenuto o internato nell'ambito della circoscrizione, si è ritenuto che tale diritto non sia sottoposto «a limitazioni o decadenze», purché la relativa richiesta sia stata presentata in modo tempestivo, «così da permettere, senza interruzioni, il regolare ed ordinato svolgimento» del procedimento di riesame. In specie, questo indirizzo giurisprudenziale ha affermato che il rinvio contenuto nell'art. 309 comma 8-bis periodo secondo c.p.p. alla richiesta formulata ai sensi del precedente comma 6 non assume carattere vincolante, sia per l'utilizzo, nel comma appena richiamato, della «formula verbale "può", in luogo di "deve", che avrebbe avuto più chiara funzione predicativa della obbligatorietà della richiesta contestuale» (del resto, non si prevede alcuna sanzione processuale espressa nel caso che quest'ultima richiesta non abbia luogo), sia per la mancanza di «statuizioni certe» su tempi e forme della richiesta di comparire all'udienza: insomma, il legislatore del 2015 avrebbe inteso concedere all'imputato in vinculis la possibilità di chiedere di comparire all'udienza «già nell'atto introduttivo dell'incidente cautelare, ma non necessariamente in concomitanza con esso».

In ultima analisi, nel bilanciamento fra l'intentio legis di individuare un momento preciso per la formulazione della richiesta di comparire all'udienza e la significatività difensiva di una simile partecipazione, l'orientamento de quo reputa che il diritto partecipativo non possa venire compresso cristallizzandone l'esercizio «in un momento - la presentazione dell'atto di riesame - che potrebbe essere ancora connotato da fluidità delle strategie difensive». Di conseguenza, la richiesta potrebbe venire formulata anche a seguito dell'avviso di fissazione dell'udienza camerale, purché - come accennato - in modo tempestivo.

Le Sezioni unite ${ }^{31}$ sono pervenute alla risoluzione della questione controversa prendendo le mosse dai capisaldi dell'orientamento

31 Cass., Sez. un., 27 febbraio 2020, n. 11803, Ramondo. Sistema penale, 24 aprile 2020, con nota critica di GRISONICH, Elisa. Il diritto dell'imputato detenuto a partecipare personalmente all'udienza di riesame: le Sezioni Unite delineano le modalità di esercizio della garanzia dopo la 1. n. 47 del 2015. Più di recente, sempre in chiave critica, v. TECCE, Raffaele. Il diritto del detenuto a partecipare all'udienza di riesame. Processo penale e giustizia, p. 1412 ss., 2020. 
giurisprudenziale maggioritario ${ }^{32}$, ma valorizzando, al contempo, la facoltà dell'imputato di chiedere personalmente il differimento dell'udienza (art. 309 comma 9-bis c.p.p.), al fine di venire sentito su specifici temi.

Nella motivazione della pronuncia viene innanzitutto compiuto un excursus della disciplina anteriore alle modifiche apportate dalla riforma del 2015, richiamando, sia pure solo a grandi linee, gli orientamenti giurisprudenziali contrapposti ${ }^{33}$ : la regolamentazione previgente emergeva, oltre che dal comma 6 dell'art. 309 c.p.p. ${ }^{34}$, dal successivo comma 8 primo periodo e dal rinvio ivi contenuto «alle forme previste dall'articolo 127» c.p.p., il cui comma 3 secondo periodo stabilisce che, se «l'interessato è detenuto o internato in luogo posto fuori dalla circoscrizione del giudice e ne fa richiesta, deve essere sentito prima del giorno dell'udienza dal magistrato di sorveglianza del luogo». Il discrimen nell'interpretazione dell'art. 127 comma 3 secondo periodo c.p.p. è senz'altro dovuto alla sentenza costituzionale n. 45 del $1991^{35}$, con la quale i giudici delle leggi decisero che la disposizione andava interpretata nel senso che il tribunale del riesame fosse tenuto ad assicurare la presenza dell'interessato davanti a sé, qualora questi avesse presentato una specifica richiesta, e il cui dictum è stato seguito da tre pronunce delle Sezioni unite ${ }^{36}$. Tuttavia, le

32 All'analisi di tale orientamento è dedicato il punto 5.1 del Considerato in diritto, mentre il punto 5.2 illustra l'indirizzo minoritario. Per un inquadramento della problematica v. GIULIANI, Livia. Art. 309. In: ILLUMINATI, Giulio; GIULIANI, Livia (diretto da). Commentario breve al codice di procedura penale. III ed. Milano: Wolters Kluver Cedam, 2020, p. 1434 s.

33 In ordine ai quali v. Cass., Sez. un., 27 febbraio 2020, n. 11803, Ramondo. Sistema penale, 24 aprile 2020, punti 3-3.3.3 del Considerato in diritto.

34 Prima dell'interpolazione operata dalla 1. n. 47 del 2015, l'art. 309 comma 6 c.p.p. stabiliva che con la richiesta di riesame «possono essere enunciati anche i motivi. Chi ha proposto la richiesta ha, inoltre, facoltà di enunciare nuovi motivi davanti al giudice del riesame facendone dare atto a verbale prima dell'inizio dell'udienza».

35 Corte cost., 17 gennaio1991-31 gennaio 1991, n. 45: si tratta di una sentenza interpretativa di rigetto, con cui la Corte dichiarò non fondata, nei sensi di cui in motivazione, la questione di legittimità del combinato disposto degli artt. 309 comma 8 e 127 comma 3 c.p.p., sollevata in riferimento all'art. 24 comma 2 Cost.

36 V., in proposito, Cass., Sez. un., 27 febbraio 2020, n. 11803, Ramondo. Sistema penale, 24 aprile 2020, punti 3.1-3.2 del Considerato in diritto, dove sono richiamate, rispettivamente, due pronunce del 1995 e del 1998 (sulle quali v. 
indicazioni fornite dalla Corte costituzionale, pur dopo il loro recepimento ad opera delle Sezioni unite, non avevano sortito l'effetto di stabilizzare la giurisprudenza, con il risultato che, in epoca prossima alla riforma del 2015, ancora si fronteggiavano orientamenti diversificati «circa la riconoscibilità - e in quali termini - del diritto dell'imputato detenuto fuori dalla circoscrizione del giudice a partecipare all'udienza di riesame $»^{37}$. Accanto all'orientamento maggioritario, il quale, allineandosi alla pronuncia costituzionale sopra ricordata, riteneva che la richiesta di essere sentito avanzata dal detenuto fuori circoscrizione fosse vincolante per il giudice del riesame, purché risultasse tempestiva rispetto al momento in cui egli aveva ricevuto la notifica dell'avviso di fissazione dell'udienza ${ }^{38}$, si registravano altresì indirizzi radicalmente negativi (che ammettevano il solo diritto a essere sentito dal magistrato di sorveglianza $)^{39} \mathrm{e}$ altri che, pur riconoscendo il diritto dell'interessato a presenziare all'udienza di riesame, ne condizionavano l'esercizio a un duplice dato contenutistico. Sarebbe stato necessario, infatti, che si versasse in ipotesi nelle quali dovessero essere esaminate questioni di fatto riguardanti la condotta dell'interessato o quest'ultimo intendesse contestare le risultanze probatorie e indicare eventualmente circostanze a sé favorevoli, anche mediante la produzione di documenti ${ }^{40}$. Si comprende pertanto che la riforma del 2015 mirasse a superare le suddette divergenze giurisprudenziali, ma lo scopo non è stato purtroppo conseguito e le difficoltà interpretative hanno propiziato ex novo le contrapposizioni già rammentate.

GIULIANI, Livia. Art. 309. In: ILLUMINATI, Giulio; GIULIANI, Livia [diretto da]. Commentario breve al codice di procedura penale. III ed. Milano: Wolters Kluver Cedam, 2020, p. 1433 s.), e una pronuncia del 2010, concernente la partecipazione al giudizio camerale di appello.

37 Cass., Sez. un., 27 febbraio 2020, n. 11803, Ramondo. Sistema penale, 24 aprile 2020, punto 3.3 del Considerato in diritto.

38 Cass., Sez. un., 27 febbraio 2020, n. 11803, Ramondo. Sistema penale, 24 aprile 2020, punto 3.3.1 del Considerato in diritto.

39 Cass., Sez. un., 27 febbraio 2020, n. 11803, Ramondo. Sistema penale, 24 aprile 2020, punto 3.3.2 del Considerato in diritto.

40 Per questo indirizzo, dunque, il giudice del riesame avrebbe avuto la facoltà di disattendere richieste formulate genericamente o a fini meramente defatigatori (cfr. Cass., Sez. un., 27 febbraio 2020, n. 11803, Ramondo. Sistema penale, 24 aprile 2020, punto 3.3.3 del Considerato in diritto). 
Prima di dare conto degli argomenti letterali e sistematici posti a fondamento della soluzione accolta dalle Sezioni unite, va posto in luce come esse abbiano puntualizzato che la disciplina sull'esercizio del diritto di comparire personalmente all'udienza di riesame si applica solo «nei confronti dell'imputato detenuto o internato ovvero sottoposto a misura coercitiva in concreto limitativa della possibilità di partecipare all'udienza», in quanto, al di fuori di questi casi, «la necessità stessa di disciplinare l'esercizio del diritto di comparizione risulterebbe del tutto irragionevole e, in buona sostanza, estranea al sistema ${ }^{41}$. Operata questa premessa sull'ambito applicativo delle disposizioni in parola, le Sezioni unite hanno considerato indispensabile tracciare un quadro dell'evoluzione, legislativa e giurisprudenziale, che ha coinvolto il procedimento cautelare in epoca successiva alla sentenza costituzionale n. 45 del 1991: la regolamentazione del «segmento della complessiva vicenda cautelare che intercorre tra l'esecuzione della misura e la richiesta di riesame consegna un assetto» grazie al quale «alla difesa è assicurata un'ampia conoscenza degli atti e degli elementi posti a sostegno dell'applicazione della misura coercitiva, conoscenza per di più stimolata dall'espressa indicazione contenuta nella comunicazione scritta destinata ad accompagnare l'ordinanza cautelare». Ed è appunto in questo quadro che si colloca «l'ancoraggio della richiesta di comparire all'udienza camerale alla presentazione della richiesta di riesame»: è fornita, «in quel momento, la conoscenza degli elementi in base ai quali operare la scelta sulla comparizione dell'imputato» ${ }^{42}$. Le Sezioni unite hanno poi aggiunto che l'art. 299 comma 3-ter c.p.p. (inserito dalla 1.8 agosto 1995, n. 332), prevedendo che, se l'istanza di revoca o di sostituzione della misura cautelare è basata su elementi nuovi o diversi da

41 Cass., Sez. un., 27 febbraio 2020, n. 11803, Ramondo. Sistema penale, 24 aprile 2020, punto 6.1 del Considerato in diritto. In dottrina aveva segnalato il problema MARZADURI, Enrico. Diritto di difesa e tempi del procedimento dinanzi al tribunale della libertà. In: GIULIANI, Livia (a cura di). La riforma delle misure cautelari personali. Torino: Giappichelli, 2015, p. 222, notando come il mancato riferimento alla condizione detentiva dell'interessato avrebbe potuto condurre, sulla scorta di una interpretazione letterale, a risultati paradossali; v. pure MAGGIO, Paola. Le impugnazioni delle misure cautelari personali. Milano: Giuffrè Francis Lefebvre, 2018, p. 210 s.

42 Cass., Sez. un., 27 febbraio 2020, Ramondo, n. 11803. Sistema penale, 24 aprile 2020, punto 7.2 del Considerato in diritto (per la disamina dell'evoluzione normativa e giurisprudenziale v. i punti 7 e 7.1 ). 
quelli già valutati, il giudice deve assumere l'interrogatorio dell'imputato che lo richieda, rappresenta «una sorta di "valvola di garanzia", che, anche dopo la definizione del procedimento incidentale di riesame, consente all'imputato», in presenza dei presupposti stabiliti, di «far valere, attraverso il contatto diretto con il giudice, le proprie ragioni» ${ }^{43}$.

Venendo agli argomenti letterali su cui le Sezioni unite hanno fondato la propria decisione, il Supremo collegio ha affermato - sulla scia dell'orientamento maggioritario - che, indipendentemente dal luogo dove il detenuto si trova, la richiesta di riesame è «la sede esclusiva» in cui l'imputato, magari per mezzo del difensore, «non trattandosi di atto personalissimo», deve esercitare il diritto di comparire personalmente ${ }^{44}$. Non convincenti sono apparse le argomentazioni utilizzate dall'orientamento minoritario per svalutare la lettera della legge: in primo luogo, la locuzione verbale «può», che compare nell'art. 309 comma 6 periodo primo c.p.p., possiede una mera «valenza ricognitiva», confermando «la collocazione del procedimento di riesame nell'ampia categoria dei procedimenti in camera di consiglio a partecipazione eventuale delle parti»; in secondo luogo, il comma 8-bis secondo periodo dell'art. 309 c.p.p. ribadisce «una correlazione in termini di contestualità tra richiesta di riesame e richiesta di comparizione personale ${ }^{45}$. Inoltre, le Sezioni unite, sulla scorta delle evidenze letterali, hanno affrontato due ulteriori questioni. Quanto alla prima, una volta riconosciuto dalla nuova normativa, in modo non equivoco, il diritto dell'interessato di comparire all'udienza camerale, anche se detenuto fuori dalla circoscrizione del giudice, ne deriva l'inapplicabilità degli artt. 309 comma 9 e 127 comma 3 (nella sola parte riferita a tale diritto) c.p.p. e 101 disp. att. c.p.p.: infatti, mantenere la possibilità di rendere dichiarazioni al magistrato di sorveglianza ${ }^{46}$ finirebbe con il

43 Cass., Sez. un., 27 febbraio 2020, n. 11803, Ramondo. Sistema penale, 24 aprile 2020, punto 7.3 del Considerato in diritto.

44 Cass., Sez. un., 27 febbraio 2020, n. 11803, Ramondo. Sistema penale, 24 aprile 2020, punto 8 del Considerato in diritto.

45 Cass., Sez. un., 27 febbraio 2020, n. 11803, Ramondo. Sistema penale, 24 aprile 2020, punto 8.1 del Considerato in diritto: le Sezioni unite hanno addotto, a conforto, che in altre disposizioni (come l'art. 666 comma 4 c.p.p.) si attribuisce al detenuto la facoltà di comparire a sua richiesta «senza specificazioni».

46 A favore di una simile possibilità v., invece, GATTO, Antonio. Il diritto dell'imputato detenuto di presenziare all'udienza di riesame al vaglio delle 
ripristinare «profili di distinzione tra le modalità di esercizio del diritto di comparizione che la nuova disciplina ha voluto superare in toto, ferma restando, naturalmente, la possibilità, sussistendone le condizioni di legge, di procedere all'esame a distanza $»^{47} e x$ art. 45 -bis disp. att. c.p.p. Quanto alla seconda, le Sezioni unite hanno negato che la necessaria contestualità fra richiesta di riesame e richiesta di comparizione personale introduca «una decadenza dalla facoltà di comparire all'udienza di riesame», dato che tale istituto presuppone la previsione e l'inosservanza di termini (art. 172 c.p.p.), assenti nella disciplina di nuovo conio. Più semplicemente, i commi 6 e 8-bis dell'art. 309 c.p.p. si limitano a disciplinare le modalità di esercizio di un diritto seguendo un modello che ne prescrive «la contestualità con una domanda o una richiesta, già previsto a proposito di altri istituti codicistici» ${ }^{4}$.

In ordine agli argomenti sistematici atti a supportare la tesi accolta, le Sezioni unite - sempre sulla falsariga dell'indirizzo maggioritario hanno ribadito la «configurazione del tutto particolare del procedimento di riesame», che si svolge «secondo una serrata scansione temporale presidiata dalla comminatoria della perdita di efficacia della misura coercitiva in caso di superamento dell'arco temporale previsto per ciascun segmento procedimentale $»^{49}$ : la contemporaneità fra richiesta di riesame e richiesta

Sezioni unite. Sistema penale, n. 2, p. 38 ss., 2020; MAGGIO, Paola. Le impugnazioni delle misure cautelari personali. Milano: Giuffrè Francis Lefebvre, 2018, p. 211; SPANGHER, Giorgio. Brevi riflessioni sistematiche sulle misure cautelari dopo la 1. n. 47 del 2015. Diritto penale contemporaneo, Rivista trimestrale, n. 4, p. 6, 2015.

47 Cass., Sez. un., 27 febbraio 2020, n. 11803, Ramondo. Sistema penale, 24 aprile 2020, punto 8.2 del Considerato in diritto: per le Sezioni unite si verifica l'incompatibilità sopravvenuta pure degli artt. 309 comma 8 e 127 comma 4 c.p.p., nella parte in cui quest'ultimo esclude il detenuto fuori circoscrizione dalla disciplina del rinvio dell'udienza per legittimo impedimento. In dottrina, nello stesso senso, MARZADURI, Enrico. Diritto di difesa e tempi del procedimento dinanzi al tribunale della libertà. In: GIULIANI, Livia (a cura di). La riforma delle misure cautelari personali. Torino: Giappichelli, 2015, p. 222 s.

48 Cass., Sez. un., 27 febbraio 2020, n. 11803, Ramondo. Sistema penale, 24 aprile 2020, punto 8.3 del Considerato in diritto, portando ad esempio l'art. 461 comma 3 c.p.p.

49 Cass., Sez. un., 27 febbraio 2020, n. 11803, Ramondo. Sistema penale, 24 aprile 2020, punto 9 del Considerato in diritto. 
di partecipare all'udienza camerale si inserisce in maniera armonica in tale contesto, perché elimina il potere discrezionale del giudice del riesame nell'individuare il carattere tempestivo della richiesta, ancorandola a un dato certo, obiettivo e incontrovertibile, e consente, sin dal momento di fissazione dell'udienza (o delle udienze, nel caso di pluralità di detenuti richiedenti), «una programmazione tendenzialmente affidabile del lavoro dei giudici del riesame», in modo da assicurare l'osservanza dei termini perentori stabiliti per la decisione e per il deposito della motivazione, «a tutela della certezza dei tempi della decisione stessa e della sua eventuale impugnazione ${ }^{50}$. L'interpretazione così delineata valorizza, secondo le Sezioni unite, l'attribuzione all'imputato della possibilità di ottenere il differimento dell'udienza a norma dell'art. 309 comma 9-bis c.p.p. ${ }^{51}$ : a

50 Cass., Sez. un., 27 febbraio 2020, n. 11803, Ramondo. Sistema penale, 24 aprile 2020, punto 9.2 del Considerato in diritto (sul profilo della programmazione del lavoro v. anche il punto 11 del Considerato in diritto).

51 Per le Sezioni unite, con l'istanza di differimento l'imputato può chiedere di «essere sentito su specifici temi (riguardanti eminentemente la quaestio facti)»; in ogni caso, i «giustificati motivi» devono attenere a «esigenze di difesa sostanziale» e non rivelarsi «meramente pretestuosi»: v. Cass., Sez. un., 27 febbraio 2020, n. 11803, Ramondo. Sistema penale, 24 aprile 2020, punto 10 del Considerato in diritto, citando Cass., sez. VI, 3 marzo 2016, n. 13050, Dall'Acqua, nella quale si è precisato che, salvo «casi di minima consistenza del materiale probatorio da consultare, tali da rendere giustificabile l'affermazione che è pretestuosa la richiesta di ritardare la decisione, il Tribunale non può sindacare la "qualità" dei motivi; non gli spetta affermare se sia adeguato o meno il termine ordinario per lo studio degli atti depositati, se sia necessario attendere il completamento delle indagini difensive, se sia opportuno consentire al nuovo difensore appena nominato di avere il tempo per una adeguata preparazione» (punto 2 del Considerato in diritto).

In altri termini, la «ragione dell'indicazione generica dei "giustificati motivi" è, quindi, il fatto che si deve solo dare atto della loro esistenza e della loro "non pretestuosità"», tenuto conto che l'art. 309 comma 9-bis c.p.p. "costituisce una eccezionale ipotesi di dilazione di un termine di decisione che è così breve ed assistito da forte sanzione processuale solo e soltanto nell'interesse del soggetto destinatario della misura cautelare ad avere una tempestiva decisione», essendo, pertanto, «ben ragionevole che al destinatario della misura venga riconosciuto il diritto ad una breve dilazione per le sue esigenze difensive» (punto 3 del Considerato in diritto; in senso conforme v. Cass., sez. II, 29 maggio 2018, n. 51932, Talevi, in tema di sequestro preventivo). Secondo le due ultime pronunce citate, l'«unico ambito di piena scelta discrezionale» riconosciuto al tribunale della libertà consiste nel valutare l'entità della dilazione temporale da cinque a dieci giorni, valutazione che potrebbe basarsi 
parere del Supremo collegio, l'istituto riconosce all'imputato la facoltà di chiedere di essere sentito nell'udienza camerale, offrendogli «uno strumento per far valere le ragioni alla base della rivalutazione - rispetto al momento della richiesta di riesame - dell'opzione relativa al suo intervento all'udienza ${ }^{52}$.

Valutando la pronuncia delle Sezioni unite, non si può fare a meno di constatare come la soluzione adottata abbia privilegiato l'esigenza di celerità del procedimento di riesame, di certo posta anch'essa a tutela dell'imputato. Allo scopo di operare un bilanciamento fra l'esigenza in discorso e il diritto del medesimo imputato a comparire nell'udienza camerale (strumento essenziale per realizzare effettivamente il diritto di difesa), le Sezioni unite hanno ancorato la richiesta de qua a quella di riesame, con una scelta che in dottrina è stata definita troppo rigida, assumendo che «sarebbe stato preferibile lasciare, in ogni caso, salva la facoltà di avanzare l'istanza di partecipazione anche con un atto distinto da quello di riesame, pur, eventualmente, formulato nella cornice temporale imposta per la proposizione dell'impugnazione ${ }^{53}$, e ripresentando così similari letture della disciplina avanzate all'indomani della novella del $2015^{54}$. Si noti, peraltro, che, tranne i casi - con ogni probabilità non

altresì su esigenze organizzative dell'ufficio: in argomento, per considerazioni critiche, v. ROMANELLI, Bartolomeo. Il differimento dell'udienza di riesame tra esigenze di difesa sostanziale e limiti all'impugnazione. Diritto penale contemporaneo, 8 giugno 2016, p. 5. Per ulteriori riferimenti giurisprudenziali v. GIULIANI, Livia. Art. 309. In: ILLUMINATI, Giulio; GIULIANI, Livia (diretto da). Commentario breve al codice di procedura penale. III ed. Milano: Wolters Kluver Cedam, 2020, p. 1433.

52 Cass., Sez. un., 27 febbraio 2020, n. 11803, Ramondo. Sistema penale, 24 aprile 2020, punto 10 del Considerato in diritto.

53 Così GRISONICH, Elisa. Il diritto dell'imputato detenuto a partecipare personalmente all'udienza di riesame: le Sezioni Unite delineano le modalità di esercizio della garanzia dopo la 1. n. 47 del 2015. Sistema penale, 24 aprile 2020.

54 Cfr. MAGGIO, Paola. Le impugnazioni delle misure cautelari personali. Milano: Giuffrè Francis Lefebvre, 2018, p. 211; MARZADURI, Enrico. Diritto di difesa e tempi del procedimento dinanzi al tribunale della libertà. In: GIULIANI, Livia (a cura di). La riforma delle misure cautelari personali. Torino: Giappichelli, 2015, p. 223 s.; MITTICA, Francesca Romana. La partecipazione all'udienza di riesame dopo la 1. n. 47 del 2015. Processo penale e giustizia, n. 3, p. 100 ss., 2016; v. inoltre, ma in termini problematici, ILLUMINATI, Giulio. Verso il 
così diffusi nella prassi, pur non esistendo dati statistici in proposito di richiesta di riesame proposta dall'indagato/imputato senza essersi consultato con il proprio difensore e priva della contestuale richiesta di comparire, in tutte le altre ipotesi, alla luce della sentenza delle Sezioni unite (che ormai costituisce un precedente per le sezioni semplici, le quali, se non condividono il principio di diritto enunciato dalle Sezioni unite, devono rimettere loro la decisione del ricorso ex art. 618 comma 1-bis c.p.p. $)^{55}$, il difensore - qualora la richiesta di riesame venga proposta non da lui bensì dall'interessato - si assicurerà che contestualmente sia presentata la richiesta di comparizione personale, alla quale d'altro canto sarà sempre possibile rinunciare ${ }^{56}$. Nelle ipotesi residuali in cui l'interessato, sprovvisto della necessaria preparazione tecnica, abbia omesso di contestualizzare la richiesta di riesame e quella di comparire personalmente, potrà venire utilizzata - su input del difensore ${ }^{57}$ - la richiesta di differimento dell'udienza ex art. 309 comma 9-bis c.p.p. ${ }^{58}$ :

ripristino della cultura delle garanzie in tema di libertà personale dell'imputato. Rivista italiana di diritto e procedura penale, p. 1157, 2015. Più di recente, MARANDOLA, Antonella. Partecipazione personale al riesame: una lettura in linea con la Costituzione. Giurisprudenza italiana, p. 1932 ss., 2019.

55 Sulla novità normativa v. il quadro tracciato da DEL COCO, Rosita. Art. 618. In: ILLUMINATI, Giulio; GIULIANI, Livia (diretto da). Commentario breve al codice di procedura penale. III ed. Milano: Wolters Kluver Cedam, 2020, p. $3050 \mathrm{~s}$.

56 Cfr. Cass., sez. I, 19 luglio 2019, n. 41935, Azzouz.

57 Per il rilievo che, «al di là della forma, l'iniziativa» ex art. 309 comma 9-bis c.p.p. «dipenda da considerazioni del difensore più che da scelte dell'assistito», v. MARZADURI, Enrico. Diritto di difesa e tempi del procedimento dinanzi al tribunale della libertà. In: GIULIANI, Livia (a cura di). La riforma delle misure cautelari personali. Torino: Giappichelli, 2015, p. 229. Secondo ILLUMINATI, Giulio. Verso il ripristino della cultura delle garanzie in tema di libertà personale dell'imputato. Rivista italiana di diritto e procedura penale, $\mathrm{p}$. 1158,2015 , la richiesta di differimento deve essere formulata personalmente dall'imputato «anche per evidenziare che la scelta non può essere determinata esclusivamente dalle esigenze professionali dell'avvocato».

58 Sul punto v. però GRISONICH, Elisa. Il diritto dell'imputato detenuto a partecipare personalmente all'udienza di riesame: le Sezioni Unite delineano le modalità di esercizio della garanzia dopo la l. n. 47 del 2015. Sistema penale, 24 aprile 2020, per la quale «l'istituto del differimento dell'udienza, circoscritto ai casi in cui l'interessato manifesti la volontà di essere sentito su specifici temi, non pare far fronte a quelle esigenze difensive» che «potrebbero 
dai responsi giurisprudenziali si evince che il rigetto di una tale richiesta si rivela oltremodo circoscritto ${ }^{59}$.

Una critica ulteriore alla pronuncia delle Sezioni unite fa leva sulla mancata considerazione del rapporto fra la normativa interna e l'art. $5 \S 4$ c.e.d.u., con riguardo alle caratteristiche che devono contraddistinguere il rimedio giurisdizionale ivi assicurato a ogni persona «privata della libertà mediante arresto o detenzione», perché lo si possa ritenere effettivo. Dalla recente giurisprudenza della Corte di Strasburgo, in un caso italiano ${ }^{60}$,

palesarsi nel corso dell'udienza camerale e non già, ed esclusivamente, al momento dell'istanza». In senso critico v. pure TECCE, Raffaele, Il diritto del detenuto a partecipare all'udienza di riesame. Processo penale e giustizia, p. 1419 s., 2020, che, de iure condendo, propone (p. 1420 s.) di prevedere la possibilità della partecipazione a distanza sull'esempio di quanto stabilisce l'art. 678 comma 3.2 c.p.p. per il detenuto o l'internato nel procedimento di sorveglianza.

59 Per l'orientamento della giurisprudenza v. supra, nota 51. Nei casi di rigetto dell'istanza di differimento, piuttosto, si prospetta il problema della non impugnabilità, data la mancanza di una specifica previsione: in proposito v. ROMANELLI, Bartolomeo. Il differimento dell'udienza di riesame tra esigenze di difesa sostanziale e limiti all'impugnazione. Diritto penale contemporaneo, 8 giugno 2016, p. 6-8.

60 Corte eur., sez. I, 5 settembre 2019, Rizzotto c. Italie ( $N^{\circ} 2$ ), §§ 50-51 e 59-60. Sebbene il caso concreto (riferito sia all'art. 309 c.p.p. sia all'art. 299 c.p.p.) concernesse un imputato latitante e presentasse quindi determinate specificità, ai nostri fini rilevano alcune affermazioni generali: in specie, la Corte ha ricordato che «la première garantie fondamentale découlant naturellement de l'article $5 \S 4$ de la Convention est le droit d'être effectivement entendu par le juge saisi d'un recours contre une détention» ( $\$ 51 \mathrm{e}$, per precedenti pronunce, $\S 46$ lett. f; v. altresì Corte eur., sez. II, 3 marzo 2020, Baş c. Turchia, §§ 212, 215, 230-231). Per commenti alla pronuncia v. CARDAMONE, Daniela. Il sistema dei rimedi de libertate alla luce della giurisprudenza della Cedu nella sentenza Rizzotto c. Italia (5 settembre 2019). Questione giustizia, $1^{\circ}$ ottobre 2019; COLAIACOVO, Guido. Latitanza e riesame (appunti a margine di una sentenza della Corte europea dei diritti dell'uomo). Cassazione penale, p. 768 ss., 2020; PRESSACCO, Luca. Latitanza e "giusto processo cautelare". Rivista italiana di diritto e procedura penale, p. 363 ss., 2020; TARALLO, Antonio. "Unicità dell'impugnazione", "giudicato cautelare" e tutela dei latitanti: qualche perplessità sulle scelte della Corte e.d.u. Processo penale e giustizia, p. 418 ss., 2020.

In certi casi la Corte di Strasburgo ha collegato la partecipazione personale dell'interessato al principio della parità delle parti (Corte eur., sez. III, 24 giugno 2004, Frommelt c. Liechtenstein, § 29; Corte eur., sez. I, 7 dicembre 2017, Stergiopoulos c. Grecia, § 48, su cui v. CASSIBBA, Fabio. Impugnazioni 
emerge, infatti, «la necessità di garantire il diritto dell'imputato di essere effettivamente sentito dal giudice», il che postulerebbe una interpretazione più flessibile rispetto a quella fatta propria dalle Sezioni unite ${ }^{61}$.

\section{Segue: misura cautelare coercitiva su appello del PUBBLICO MINISTERO (ART. 310 COMMI 1 E 3 C.P.P.) E INTERROGATORIO DI GARANZIA}

Un contrasto interpretativo di non particolare consistenza numerica $^{62}$ si è verificato nella situazione in cui il tribunale della libertà, operando come giudice di appello adito dal pubblico ministero che lamenta il rigetto della propria richiesta di misura cautelare coercitiva da parte del

de libertate e garanzie minime dell'equità processuale. Rivista italiana di diritto e procedura penale, p. 968-970, 2018), mentre in altre occasioni ha posto l'accento sulla conoscenza diretta dei fatti da parte del detenuto (Corte eur., sez. I, $1^{\circ}$ giugno 2006, Mamedova c. Russia, § 91). Tuttavia, talora la Corte europea ha affermato che nell'udienza ove si decide l'impugnazione cautelare il diritto di difesa può venire tutelato ascoltando le ragioni dell'interessato «either in person or through some form of representation» (Corte eur., Grande Camera, 22 maggio 2012, Idalov c. Russia, § 161).

Quanto all'art. 6 c.d.f.u.e. («Ogni persona ha diritto alla libertà e alla sicurezza»), secondo la Spiegazione relativa a quest'ultimo (leggibile in NASCIMBENE, Bruno [a cura di]. Unione europea. Trattati. Torino: Giappichelli, 2010, p. 381 s.) i diritti in esso garantiti corrispondono a quelli garantiti dall'art. 5 c.e.d..u., e, ai sensi dell'art. $52 \S 3$ della Carta, «hanno pari significato e portata».

61 In questa direzione v. GRISONICH, Elisa. Il diritto dell'imputato detenuto a partecipare personalmente all'udienza di riesame: le Sezioni Unite delineano le modalità di esercizio della garanzia dopo la 1. n. 47 del 2015. Sistema penale, 24 aprile 2020, a parere della quale l'accoglimento della soluzione propugnata dall'indirizzo minoritario «avrebbe riconsegnato un assetto legislativo (...) più rispondente ai principi costituzionali e convenzionali»; cfr. TECCE, Raffaele, Il diritto del detenuto a partecipare all'udienza di riesame, in Processo penale e giustizia, p. 1420, 2020.

62 Come emerge dall'ordinanza di rimessione alle Sezioni unite (Cass., sez. VI, ord. 18 dicembre 2019, n. 1243, Salvati, punti 2 e 3 del Considerato in diritto), il contrasto è infatti sorto inizialmente all'interno della stessa sesta sezione, che, dopo avere in una prima pronuncia sostenuto l'orientamento restrittivo, in una successiva decisione ha aderito a quello più ampio. L'indirizzo restrittivo è stato poi accolto da Cass., sez. II, 25 maggio 2017, n. 38828, Savina. 
giudice per le indagini preliminari, applichi tale misura: il punctum dolens da risolvere è se, in una evenienza del genere, vada effettuato oppure no l'interrogatorio di garanzia ex art. 294 c.p.p.

Secondo l'indirizzo giurisprudenziale più restrittivo ${ }^{63}$, non è necessario procedere all'interrogatorio in discorso perché «il provvedimento emesso in sede di appello cautelare è preceduto dall'instaurazione di un contraddittorio pieno, finalizzato ad approfondire anticipatamente tutti i temi dell'azione cautelare anche attraverso i contributi forniti dalla difesa»: sussiste quindi una inversione rispetto all'ordinaria ipotesi nella quale il contraddittorio risulta differito in rapporto alla misura cautelare già disposta. In quest'ottica, dunque, imporre l'interrogatorio di garanzia dopo l'applicazione della misura coercitiva in sede di appello cautelare si tradurrebbe, addirittura, in una «superfetazione difensiva», trasformando l'atto de quo in «una formalità superflua, ampiamente assorbita dalla dinamica dell'attività processuale che la precede».

Per la tesi giurisprudenziale più ampia ${ }^{64}$, viceversa, l'interrogatorio di garanzia si connota come imprescindibile (a meno che sia già iniziata la fase dibattimentale), e di conseguenza il suo mancato o tardivo espletamento comporta la perdita di efficacia della misura cautelare. A sostegno si sono innanzitutto addotti gli artt. 294 e 302 comma 1 primo periodo c.p.p., dai quali emerge come il giudice che abbia emesso un provvedimento limitativo della libertà personale deve interrogare la persona sottoposta alla misura, a pena di inefficacia di quest'ultima, a meno che all'interrogatorio si sia già proceduto in sede di convalida della misura precautelare oppure che sia iniziato il dibattimento, dove l'imputato può chiedere di essere sottoposto a interrogatorio (rectius, esame), nel contraddittorio fra le parti. Ne deriva che, non essendo prevista alcuna eccezione per l'ipotesi in cui il provvedimento cautelare sia stato emesso dal tribunale della libertà a seguito di appello del pubblico ministero avverso l'ordinanza reiettiva del giudice per le indagini preliminari, conserva validità la regola generale; sulla stessa linea si colloca, del resto,

63 In proposito v. Cass., sez. VI, 18 dicembre 2019, n. 1243, Salvati, punti 2-2.2 del Considerato in diritto, ai quali si riferiscono le citazioni riportate nel testo.

64 Per le argomentazioni a fondamento di questa tesi v. Cass., sez. VI, 18 dicembre 2019, n. 1243, Salvati, punti 3-3.4 del Considerato in diritto, cui rimandano le citazioni che compaiono nel testo. 
pure l'art. 302 comma 1 secondo periodo c.p.p., che, nell'eventualità di caducazione della misura cautelare per omesso o intempestivo interrogatorio ai sensi dell'art. 294 c.p.p., impone «la rinnovazione dell'interrogatorio a piede libero, a conferma dell'assoluta inderogabilità dell'incombente processuale, pena l'inapplicabilità del provvedimento coercitivo». Si è al contempo rimarcato che l'interrogatorio di garanzia non ha una «finalità istruttoria», bensì «squisitamente difensiva», mirando a consentire al giudice di «verificare, alla luce delle stesse parole della persona assoggettata a vincolo, la sussistenza ab origine e la persistenza, in quel momento, delle condizioni di applicabilità della misura». Di specifica pregnanza l'osservazione che l'interrogatorio di garanzia non può essere surrogato dalla facoltà della persona interessata di rendere dichiarazioni spontanee nel corso dell'udienza dell'appello cautelare: molteplici, infatti, i discostamenti fra i due atti che li rendono «ontologicamente diversi», senza contare che le dichiarazioni spontanee intervengono quando «la cautela personale non è in atto e costituisce solo un'ipotesi, seppure concreta e da contrastare», mentre nell'interrogatorio di garanzia la persona è chiamata a difendersi in relazione «ad un titolo coercitivo già in esecuzione e, dunque, concreto». Infine, si è rilevato che, nella prassi, fra l'applicazione della misura cautelare da parte del tribunale della libertà a norma dell'art. 310 c.p.p. e la sua effettiva esecuzione può frapporsi un lasso di tempo durevole ${ }^{65}$ : il pressoché scontato ricorso per cassazione

65 L'art. 311 comma 5 c.p.p. stabilisce che la «corte di cassazione decide entro trenta giorni dalla ricezione degli atti», osservando le forme previste dall'art. 127 c.p.p.: ma il termine di trenta giorni ha carattere ordinatorio e i dati statistici relativi all'anno 2019 documentano che la durata media (dall'iscrizione in cancelleria penale all'udienza) dei ricorsi definiti in tema di misure cautelari personali corrisponde a 63 giorni (v. Corte Suprema di Cassazione, Ufficio di statistica, La Cassazione penale - Annuario statistico 2019, Tab. 5.2). In caso di annullamento con rinvio, inoltre, possono verificarsi dei "tempi morti" fra la decisione della Suprema Corte e la trasmissione degli atti al giudice di rinvio e bisogna poi calcolare la (innovata) tempistica del giudizio di rinvio (v. infra, § 4 e nota 109). Quanto all'ammontare dei ricorsi, dalla Tab. 3.1 emerge che nel 2019 sono stati iscritti 4.166 ricorsi in materia di misure cautelari personali, pari all' $8,2 \%$ del totale dei procedimenti iscritti (50.801) e dalla Tab. 4.7 risulta che ne sono stati definiti 4.188 , pari all' $8,1 \%$ del totale dei procedimenti definiti (51.420). Nel 2020 la durata media è salita a 89 giorni (v. Corte Suprema di Cassazione, Ufficio di statistica, La Cassazione penale - Annuario statistico 2020, Tab. 6.2), ma l'aumento dipende 
ex art. 311 c.p.p. produce, infatti, la sospensione dell'esecuzione della decisione finché quest'ultima non sia divenuta definitiva (art. 310 comma 3 c.p.p.), e pertanto solo l'interrogatorio dopo l'esecuzione della misura cautelare può garantire il controllo del giudice sulla persistenza delle condizioni di applicabilità della misura stessa.

L'ordinanza di rimessione ha considerato preferibile il secondo orientamento $^{66}$ : oltre a ribadire i capisaldi sui quali esso si fonda, in particolare definendo "non superabile» ${ }^{67}$ il rilievo secondo cui l'interrogatorio di garanzia non può venire surrogato dalla facoltà della persona di rendere dichiarazioni spontanee nell'udienza di discussione dell'appello cautelare, l'ordinanza si è soffermata sulle argomentazioni avanzate dai giudici costituzionali in ormai risalenti pronunce che hanno dichiarato la parziale illegittimità degli artt. 294 comma 1 e 302 c.p.p. ${ }^{68}$. Tirando le somme, la Corte costituzionale ha ritenuto che la conoscenza degli atti delle indagini preliminari, la possibilità di fornire elementi a proprio favore tramite la difesa tecnica e la facoltà per l'indagato/imputato di essere sentito dal tribunale del riesame non eliminano la necessità dell'interrogatorio di garanzia: perciò, la doverosità di tale interrogatorio sussiste, per identità di ratio, anche se la misura cautelare sia stata emessa in sede di appello proposto dal pubblico ministero ex art. 310 c.p.p. ${ }^{69}$. Conclusione alla quale non è d'ostacolo la circostanza che l'art. 294 comma 1 c.p.p. individui come competente a compiere l'interrogatorio di garanzia il giudice «che ha deciso in ordine all'applicazione della

esclusivamente dalla sospensione processuale disposta ex lege a causa della pandemia da Covid-19 (CURZIO, Piero. Relazione sull'amministrazione della giustizia nell'anno 2020. Roma, 29 gennaio 2021, p. 73). Dai dati statistici si ricava che nell'anno 2020 sono stati iscritti (Tab. 2.1) 3.947 ricorsi concernenti le misure cautelari personali, pari al 10,2\% del totale dei procedimenti iscritti (38.508) e ne sono stati definiti (Tab. 5.6) 3.738, pari al 10,0\% del totale dei procedimenti definiti (37.344).

Orientamento che, come si è detto (v. supra, nota 62), la sesta sezione aveva già abbracciato (Cass., sez. VI, 20 novembre 2014, n. 6088, Lo Nardo).

67 Cass., sez. VI, 18 dicembre 2019, n. 1243, Salvati, punto 5.1 del Considerato in diritto.

68 Cfr. Corte cost., 24 marzo 1997-3 aprile 1997, n. 77 e Corte cost., 10 febbraio 1999-17 febbraio 1999, n. 32.

69 Cass., sez. VI, 18 dicembre 2019, n. 1243, Salvati, punto 5.5 del Considerato in diritto. 
misura cautelare», in quanto la giurisprudenza della Suprema Corte si è già espressa nel senso che - nella situazione in oggetto - competente a procedere all'interrogatorio è sempre il giudice per le indagini preliminari ${ }^{70}$.

Le Sezioni unite ${ }^{71}$, all'opposto, hanno aderito all'interpretazione restrittiva, assumendo che la tesi sostenuta nell'ordinanza di rimessione, pur utilizzando «argomenti suggestivi», basati sulla valorizzazione dell'interrogatorio come momento fondante del diritto di difesa, «riguarda situazioni specifiche estranee» a quella che viene in gioco. A detta del Supremo consesso, infatti, l'art. 294 c.p.p. (come riletto dalla Corte costituzionale) «non è esportabile» tout court alla fattispecie della misura adottata in sede di appello cautelare, perché le finalità difensive sono tutelate dal contraddittorio che si esplica nel procedimento camerale, non rilevando, d'altra parte, la facoltatività delle dichiarazioni spontanee, bensì la circostanza che l'interessato sia "posto nelle condizioni di esercitare appieno le proprie difese» ${ }^{72}$, essendone riservato il quomodo alla sua scelta discrezionale. Ad adiuvandum, le Sezioni unite hanno addotto che la 1. n. 47 del 2015 ha allargato la possibilità di partecipazione personale dell'indagato alla fase dell'impugnazione cautelare, richiamando l'art. 309 comma 6 c.p.p. e il relativo diritto di comparire personalmente all'udienza di riesame ${ }^{73}$ : ma (si ritornerà sul punto più avanti), in realtà, tale diritto non è estensibile all'udienza dell'appello cautelare, visto che il comma 6 dell'art. 309 c.p.p. non è ricompreso tra le disposizioni da osservare alle quali l'art. 310 comma 1 primo periodo c.p.p. rinvia $^{74}$.

70 Cass., sez. VI, 18 dicembre 2019, n. 1243, Salvati, punto 6.1 del Considerato in diritto, cui si rinvia per gli opportuni richiami giurisprudenziali.

71 Cass., Sez. un., 26 marzo 2020, n. 17274, Salvati. Sistema penale, 15 giugno 2020, con nota adesiva di DE ROBBIO, Costantino. Applicazione di misura cautelare da parte del tribunale del riesame e interrogatorio di garanzia: la sentenza delle Sezioni unite. Per rilievi critici v., invece, MARANDOLA, Antonella. Misura cautelare applicata dal tribunale della libertà in sede d'appello: negato l'interrogatorio di garanzia. Cassazione penale, p. 4038 ss., 2020; PERONI, Francesco. Davvero superfluo l'interrogatorio di garanzia in caso di cautela adottata in sede d'appello?. Diritto penale e processo, p. 65 ss., 2021.

72 Cass., Sez. un., 26 marzo 2020, n. 17274, Salvati. Sistema penale, 15 giugno 2020, punto 4 del Considerato in diritto, anche per la precedente citazione.

73 V. ancora Cass., Sez. un., 26 marzo 2020, n. 17274, Salvati. Sistema penale, 15 giugno 2020, punto 4 del Considerato in diritto.

74 V. supra, $\S 1$, nel testo e nota 19. 
L'architrave su cui poggia il ragionamento delle Sezioni unite è che la disciplina dell'interrogatorio di garanzia stabilita dall'art. 294 c.p.p., certamente «essenziale in taluni contesti procedimentali», non è sempre «ineliminabile e insostituibile per l'esercizio pieno delle facoltà defensionali» ${ }^{75}$. Accanto a situazioni, assimilabili a quella colà regolata, dove tale interrogatorio è presente (artt. 299 comma 3-ter e 302 c.p.p.), se ne annoverano altre nelle quali l'interrogatorio è invece previsto prima dell'adozione della misura (art. 289 c.p.p. e art. 47 d.lgs. 8 giugno 2001, n. 231, in tema di responsabilità amministrativa degli enti), avendo il legislatore inteso «valorizzare un momento di conoscenza anticipata delle ragioni difensive, a fronte della potenziale "invasività" qualitativa» della misura stessa, e altre ancora - vi appartiene quella che si sta esaminando - in cui l'interrogatorio non è previsto e non è necessario poiché «il contraddittorio è veicolato in altra equipollente maniera» ${ }^{76}$.

Con particolare riferimento a quest'ultima tipologia di ipotesi, le Sezioni unite hanno portato ad esempio, da un canto, la custodia cautelare disposta dopo la sentenza di condanna e, dall'altro, la sostituzione o il cumulo della misura trasgredita con altra più grave (art. 276 c.p.p.). Nella prima eventualità, la mancanza dell'interrogatorio di garanzia non priva l'imputato del suo pieno diritto di difesa perché lo svolgimento della fase dibattimentale gli ha consentito di «prospettare al giudice tutte le ragioni difensive, anche attraverso l'esame o le dichiarazioni spontanee»; nella seconda, l'interrogatorio si è già svolto in occasione della misura applicata $a b$ origine e poi trasgredita, mentre la sostituzione o il cumulo conseguono alla ritenuta violazione delle prescrizioni collegate alla misura, "attaccabile" con l'impugnazione del provvedimento ${ }^{77}$.

In conclusione, le Sezioni unite hanno sottolineato che appunto «la diversità di meccanismi procedimentali» atti a garantire il diritto di difesa

75 Cass., Sez. un., 26 marzo 2020, n. 17274, Salvati. Sistema penale, 15 giugno 2020, punto 5.1 del Considerato in diritto.

76 Cass., Sez. un., 26 marzo 2020, n. 17274, Salvati. Sistema penale, 15 giugno 2020, punto 5.1 del Considerato in diritto. Per una disamina delle prime due categorie di situazioni v., rispettivamente, punti 6 e 7 del Considerato in diritto.

77 Cass., Sez. un., 26 marzo 2020, n. 17274, Salvati. Sistema penale, 15 giugno 2020, punto 8 del Considerato in diritto. 
conferma la non estensibilità dell'interrogatorio disciplinato nell'art. 294 c.p.p., qualora si rifletta che «il provvedimento emesso in sede di appello cautelare è preceduto da un contraddittorio pieno, finalizzato ad anticipare tutti i temi dell'azione cautelare, anche attraverso i contributi forniti dalla difesa», e che al giudice possono venire prospettate «le ragioni a supporto dell'auspicato diniego della richiesta cautelare del pubblico ministero». Anzi, «una semplicistica estensione dell'obbligo di interrogatorio» si tradurrebbe in «un vuoto formalismo» e, in chiusura, le Sezioni unite giungono a domandarsi «quale significato e valenza potrebbe avere» la previsione di un interrogatorio che «dovrebbe espletare, di fatto, almeno nella fase delle indagini, quello stesso giudice che, in prima battuta, ha negato l'applicabilità della misura ${ }^{78}$.

La soluzione sposata dalle Sezioni unite non appare convincente per varie ragioni, alcune ben evidenziate dalla stessa ordinanza di rimessione. Va innanzitutto sgombrato il campo - lo si è anticipato - dall'assunto che la facoltà di rendere dichiarazioni spontanee nell'udienza di appello cautelare sia divenuta oggi più intensa sulla base dell'art. 309 comma 6 primo periodo c.p.p., a norma del quale con la richiesta di riesame «l'imputato può chiedere di comparire personalmente»: la disposizione (analogamente al comma 8-bis secondo periodo del medesimo art. 309 c.p.p.) non è applicabile all'appello cautelare, non essendo richiamata dall'art. 310 comma 2 primo periodo c.p.p..$^{79}$; continua perciò a valere l'art. 127 comma 3 secondo periodo c.p.p., in virtù del rinvio alle forme previste da tale ultimo articolo operato dall'art. 310 comma 2 terzo periodo c.p.p. ${ }^{80}$.

78 Cass., Sez. un., 26 marzo 2020, n. 17274, Salvati. Sistema penale, 15 giugno 2020, punto 9 del Considerato in diritto.

79 Nel senso del testo v. VARONE, Fabio. A proposito della fungibilità delle garanzie: le Sezioni unite negano il diritto dell'accusato all'interrogatorio in caso di applicazione delle misure coercitive a seguito di appello del pubblico ministero. Archivio penale (web), n. 2, p. 12, nota 30, 2020. CASSIBBA, Fabio. Impugnazioni de libertate e garanzie minime dell'equità processuale. Rivista italiana di diritto e procedura penale, p. 971, 2018, osserva che, nel valutare la compatibilità costituzionale degli artt. 310 comma 2 e 127 comma 3 c.p.p. sotto l'aspetto illustrato, «occorrerebbe, almeno, distinguere fra le ipotesi in cui vengono in gioco questioni di merito e quelle in cui vengono in gioco questioni di puro diritto».

80 Su tale rinvio v. PERONI, Francesco. Davvero superfluo l'interrogatorio di garanzia in caso di cautela adottata in sede d'appello?. Diritto penale e processo, 
Ciò premesso, paiono in effetti difficili da superare i divari tra dichiarazioni spontanee e interrogatorio di garanzia, che rendono i due atti non surrogabili ${ }^{81}$ : oltre alla loro ontologica differenza, vanno enumerate la mera eventualità delle dichiarazioni spontanee, «le eterogenee modalità di espletamento (giusta l'assenza di uno scambio "domanda e risposta" e, soprattutto, di una contestazione degli elementi a carico, che contraddistinguono l'interrogatorio), la diversità dei tempi dedicabili all'uno o all'altro segmento processuale». Si aggiunga che la situazione in cui viene a trovarsi l'indagato nell'udienza di discussione dell'appello cautelare (allorché la restrizione della sua libertà è solo ipotetica) non è certo paragonabile a quella in cui egli versa dopo l'emissione del provvedimento cautelare da parte del tribunale della libertà, operante come giudice di appello, a titolo esecutivo già eseguito ${ }^{82}$.

Pur riconoscendo che di solito il titolo non viene immediatamente eseguito perché la proposizione del ricorso per cassazione ex art. 311 c.p.p. ne sospende l'esecuzione (art. 310 comma 3 c.p.p.), le riserve sulla soluzione patrocinata dalle Sezioni unite non sfumano: è vero che, in questo caso, la libertà personale non è compressa, tuttavia non rappresenta la soluzione migliore limitarsi ad attendere la sentenza definitiva della Corte di cassazione (destinata a intervenire a vari mesi di distanza), che non può comunque avere effettuato un controllo di merito sulla legalità della misura cautelare, con evidenti frizioni sul piano dell'art. 5 \& 4 c.e.d.u. ${ }^{83}$.

p. 66, 2021, dove rileva come, traducendo «il rinvio in dato normativo, ne emerge che, in sede di appello cautelare, l'indagato/imputato è "sentito" e non "interrogato": espressioni, all'evidenza, non riducibili a sinonimo».

81 In proposito v. GREVI, Vittorio; CERESA-GASTALDO, Massimo. Misure cautelari. In: BARGIS, Marta (a cura di). Compendio di procedura penale, $\mathrm{X}$ ed. Milano: Wolters Kluver Cedam, 2020, p. 372; PERONI, Francesco. Davvero superfluo l'interrogatorio di garanzia in caso di cautela adottata in sede d'appello?. Diritto penale e processo, p. 66, 2021.

Cass., sez. VI, 18 dicembre 2019, n. 1243, Salvati, punto 5.1 del Considerato in diritto, altresì per la precedente citazione.

${ }_{83}$ Sul punto v. VARONE, Fabio. A proposito della fungibilità delle garanzie: le Sezioni unite negano il diritto dell'accusato all'interrogatorio in caso di applicazione delle misure coercitive a seguito di appello del pubblico ministero. Archivio penale (web), n. 2, p. 15 s., 2020. 
Per qualcuno ${ }^{84}$ l'interrogatorio di garanzia risponderebbe a due esigenze: permettere alla persona interessata di difendersi nel merito sulle ragioni che hanno supportato il provvedimento emesso in sede di appello cautelare; consentire a un giudice, diverso da quello che ha adottato la misura, di rivalutarne i presupposti applicativi, «anche alla luce del tempo trascorso tra la decisione dell'appello e la sua definitività», che può determinare «un'incidenza notevole sul permanere delle esigenze cautelari», da verificare al momento dell'effettiva esecuzione della misura. Sebbene non possa sottacersi - come da altri si è osservato - che, «in siffatto scenario, il dies a quo, imposto dall'art. 294 c.p.p. a far tempo dall'esecuzione del provvedimento de libertate, si troverebbe a decorrere ben oltre il fisiologico, con inesorabile sacrificio di quella prossimità cronologica, atta ad assicurare, nella dinamica ordinaria, l'efficienza del contraddittorio e l'efficacia del controllo giudiziale» ${ }^{85}$. Escluso che sia

84 Cfr. VARONE, Fabio. A proposito della fungibilità delle garanzie: le Sezioni unite negano il diritto dell'accusato all'interrogatorio in caso di applicazione delle misure coercitive a seguito di appello del pubblico ministero. Arch. pen. (web), n. 2, p. 16, 2020; URBINATI, Francesco. Interrogatorio di garanzia e misure cautelari: configurazioni processuali. Archivio penale (web), n. 2, p. 22, 2020, il quale ritiene opportuno un intervento legislativo che preveda «la obbligatorietà dell'interrogatorio di garanzia per la prima applicazione di misura cautelare in fase di appello, così colmando una vacatio di tutela di notevole rilievo»; per identica conclusione ARASI, Simona. L'interrogatorio di garanzia in caso di applicazione di misura cautelare coercitiva da parte del Tribunale del riesame in accoglimento dell'appello del Pubblico Ministero: obbligo o facoltà?. Processo penale e giustizia, p. 93, 2021. In senso critico v. inoltre MARANDOLA, Antonella. Misura cautelare applicata dal tribunale della libertà in sede d'appello: negato l'interrogatorio di garanzia. Cassazione penale, p. 4039, 2020, dove si sottolinea come non possa «essere trascurato il rilievo che l'atto di garanzia è forma di tutela "personale" del soggetto sottoposto alla misura, non surrogabile con l'esercizio della difesa tecnica, ma espressione dell'autodifesa». Qualche riserva, sebbene in un quadro di generale adesione alla pronuncia delle Sezioni unite, avanza pure DE ROB$\mathrm{BIO}$, Costantino. Applicazione di misura cautelare da parte del tribunale del riesame e interrogatorio di garanzia: la sentenza delle Sezioni unite. Sistema penale, 15 giugno 2020.

85 PERONI, Francesco. Davvero superfluo l'interrogatorio di garanzia in caso di cautela adottata in sede d'appello?. Diritto penale e processo, p. 66 s., 2021, secondo il quale si tratta della «contraddizione con cui fatalmente viene a scontrarsi la soluzione interpretativa più aderente, insieme, all'intelaiatura del codice e alla tutela del contraddittorio nell'incidente cautelare». 
percorribile «il tentativo di ritenere integrato il contraddittorio ogni qual volta, nel contesto dell'udienza, l'interessato sia stato sentito con modalità dialettiche assimilabili a quelle tipiche dell'interrogatorio di garanzia: vale a dire nell'osservanza delle forme di cui agli artt. 64 e 65 c.p.p. ${ }^{86}$, si è auspicato un intervento legislativo che introduca una deroga espressa all'interrogatorio di garanzia «ogni qual volta il tribunale della libertà vi abbia proceduto, nelle forme rituali, prima di adottare la cautela all'esito dell'udienza ex art. 310 c.p.p.» ${ }^{87}$.

Certo è, comunque, che nella vicenda risolta dalle Sezioni unite, al bivio tra esigenze di celerità e diritto di difesa dell'indagato, il vulnus ha inficiato il secondo termine del binomio.

\section{SEgue: GIUdizio di RIESAME IN SEDE DI RINVIO E DECORRENZA DEL TERMINE DI DIECI GIORNI DALLA «RICEZIONE DEGLI ATTI» PER LA DECISIONE (ART. 311 COMMA 5-BIS C.P.P.)}

L'introduzione nell'art. 311 c.p.p. del nuovo comma 5-bis - sul quale ci siamo già soffermati ${ }^{88}$ - ha condotto per tabulas al superamento di quell'indirizzo giurisprudenziale che, in epoca anteriore alla riforma del 2015, riteneva inoperanti nel giudizio di rinvio davanti al tribunale della libertà i termini perentori stabiliti nell'art. 309 comma 10 c.p.p. per il giudizio di riesame ${ }^{89}$. Come si è puntualizzato in dottrina, l'idea alla

86 PERONI, Francesco. Davvero superfluo l'interrogatorio di garanzia in caso di cautela adottata in sede d'appello?. Diritto penale e processo, p. 67, 2021, pone l'accento soprattutto sulla «probabile controvertibilità della pretesa metamorfosi delle dichiarazioni spontanee in interrogatorio, passibile di letture contrapposte e, in definitiva, di una perniciosa instabilità dell'epilogo decisorio de libertate».

PERONI, Francesco. Davvero superfluo l'interrogatorio di garanzia in caso di cautela adottata in sede d'appello?. Diritto penale e processo, p. 67, 2021.

Cfr. supra, § 1 .

In proposito v. BARGIS, Marta. Le novità nella disciplina del giudizio di rinvio: una replica imperfetta. Disponibile in: http://www.lalegislazionepenale. eu, 22 settembre 2015, p. 7 s. (v. pure p. 13 s., per l'assunto che il nuovo comma 5-bis dell'art. 311 c.p.p. è applicabile anche se l'ordinanza che ha disposto la misura coercitiva sia stata emessa in sede di appello cautelare attivato dal pubblico ministero avverso il rigetto della propria richiesta; in senso 
base di un simile indirizzo, cioè quella che nel giudizio di rinvio non si ponessero problemi di urgenza nell'assumere la decisione, si scontrava con il principio di inviolabilità della libertà personale, non appena si considerasse che dopo la pronuncia di annullamento, seppure con rinvio, del provvedimento restrittivo veniva «a mancare un titolo valido atto a giustificare la limitazione della libertà personale» e che l'esigenza di tempestività della decisione sussisteva in re ipsa, essendo «già trascorsi periodi significativi dall'inizio della coercizione personale» ${ }^{90}$.

Se, dunque, questo aspetto della riforma non può che essere apprezzato, la formulazione del comma in oggetto ha dato luogo - per quel che qui importa - a un contrasto interpretativo rimesso alle Sezioni unite $^{91}$, vertente sul significato della locuzione «ricezione degli atti», da cui inizia a decorrere il termine perentorio di dieci giorni per la decisione. L'orientamento ab initio formatosi reputava che la locuzione de qua andasse riferita alla ricezione da parte del giudice di rinvio degli atti nuovamente richiesti dal presidente del tribunale all'autorità giudiziaria procedente, posto che anche la decisione da adottare in tale giudizio - al pari di quella nel giudizio ordinario di riesame - presuppone sia la disponibilità di tutti gli atti presentati dal pubblico ministero a fondamento della richiesta di applicazione della misura cautelare sia l'esame degli elementi eventualmente sopravvenuti nel frattempo ${ }^{92}$. Interveniva poi una pronuncia di segno contrario ${ }^{93}$, secondo la quale il termine per la decisione sarebbe

conforme MARZADURI, Enrico. Diritto di difesa e tempi del procedimento dinanzi al tribunale della libertà. In: GIULIANI, Livia [a cura di]. La riforma delle misure cautelari personali. Torino: Giappichelli, 2015, p. 247 s.).

90 Per questi rilievi MARZADURI, Enrico. Diritto di difesa e tempi del procedimento dinanzi al tribunale della libertà. In: GIULIANI, Livia (a cura di). La riforma delle misure cautelari personali. Torino: Giappichelli, 2015, p. 245 s.; cfr. ILLUMINATI, Giulio. Verso il ripristino della cultura delle garanzie in tema di libertà personale dell'imputato. Rivista italiana di diritto e procedura penale, p. 1160, 2015.

91 Cfr. Cass., sez. VI, ord. 16 gennaio 2020, n. 4125, Calella. Sistema penale, 15 luglio 2020, con nota di ALONZI, Fabio. L'importanza della celerità per l'assunzione delle decisioni de libertate.

92 Per la necessaria ricostruzione v. Cass., sez. VI, ord. 16 gennaio 2020, n. 4125, Calella. Sistema penale, 15 luglio 2020, punto 4 del Considerato in diritto.

93 Cass., sez. I, 29 gennaio 2018, n. 23707, Battaglia, sulle cui argomentazioni v. Cass., sez. VI, ord. 16 gennaio 2020, n. 4125, Calella. Sistema penale, 15 luglio 
decorso dal momento in cui perviene al tribunale il fascicolo inoltrato dalla Corte di cassazione con la sentenza rescindente: nell'art. 311 comma 5-bis c.p.p. non compare, infatti, alcun espresso riferimento a una nuova richiesta degli atti all'autorità giudiziaria procedente, che corrisponda a quella prevista nell'art. 309 comma 5 c.p.p. per il giudizio di riesame, lettura, quest'ultima, corroborata da due considerazioni. Innanzitutto, essa sarebbe coerente con l'art. 623 lett. a c.p.p., a norma del quale l'annullamento dell'ordinanza impugnata comporta la trasmissione degli atti al giudice che l'ha pronunciata, con la conseguenza che la disponibilità degli atti per il giudice di rinvio sarebbe assicurata dalla loro presenza nel fascicolo trasmesso dalla Suprema Corte. In secondo luogo, andrebbe mutuato da una pronuncia delle Sezioni unite, attinente ad altro aspetto controverso dell'art. 311 comma 5-bis c.p.p. ${ }^{94}$, il principio di una rigorosa interpretazione del comma in oggetto, che predispone per la fase di rinvio una disciplina specifica, da considerare autonoma rispetto a quella dettata per il giudizio di riesame: in quest'ottica, la mancanza di un rimando alla richiesta di trasmissione degli atti all'autorità procedente esprimerebbe la volontà legislativa di escluderla nel giudizio di rinvio.

In seguito, peraltro, si è riproposto il pregresso orientamento senz'altro maggioritario - che, nel ribadire l'assunto di base, ha altresì sottoposto a critica la pronuncia che se ne era discostata ${ }^{95}$ : quanto al richiamo all'art. 623 lett. a c.p.p., è stato giudicato inconferente, dovendosi tenere conto della natura incidentale del procedimento di riesame e della non disponibilità del fascicolo da parte del giudice di rinvio (non

2020, punti 2-2.4 del Considerato in diritto.

94 Il riferimento è a Cass., Sez. un., 20 luglio 2017, n. 47970, Rezmuves. Processo penale e giustizia, p. 254, 2018. In senso adesivo v. ALONZI, Fabio. Un richiamo al rigore nell'interpretazione delle norme de libertate. Processo penale e giustizia, p. 259 ss., 2018. Con tale decisione, le Sezioni unite hanno affermato che il deposito in cancelleria dell'ordinanza del giudice del rinvio deve avvenire entro trenta giorni dalla decisione e non nel più lungo termine, comunque non eccedente il quarantacinquesimo giorno, previsto dall'art. 309 comma 10 c.p.p.

95 V., in specie, Cass., sez. II, 26 giugno 2019, n. 31281, Montante. Sistema penale, 21 gennaio 2020, con nota favorevole di COLAIACOVO, Guido. I tempi del giudizio di rinvio in materia cautelare (a margine di una recente sentenza della Suprema Corte). 
esistendo disposizioni che prevedano di trattenere gli atti presso il tribunale del riesame in pendenza del giudizio di cassazione), cui non può sopperire la restituzione degli atti ad opera della Corte di cassazione, visto che l'art. 100 disp. att. c.p.p. dispone l'invio alla Suprema Corte, con il ricorso, solo degli atti necessari per la decisione sull'impugnazione, non sempre coincidenti con tutti gli atti valutati ai fini della decisione poi annullata con rinvio. Anche il raccordo con la sentenza delle Sezioni unite Rezmuves è stato svalutato, e si è concluso che l'art. 311 comma 5-bis c.p.p., occupandosi solo del «segmento conclusivo» del giudizio di rinvio, non interviene sulle precedenti «scansioni procedurali», conseguendone che la disciplina descritta nell'art. 309 comma 5 c.p.p. si estende alla fase del giudizio di rinvio.

Le Sezioni unite ${ }^{96}$ hanno aderito all'orientamento prevalente, muovendo dal rilievo che appare centrale risolvere il problema relativo «all'accertamento della necessità, per il corretto svolgimento del giudizio di rinvio nel procedimento di riesame, di una nuova richiesta di atti all'autorità procedente», in virtù di «un implicito richiamo» a quanto disposto dall'art. 309 comma 5 c.p.p. per il giudizio ordinario di riesame ${ }^{97}$. Da questo angolo visuale, i giudici di legittimità hanno premesso che l'interpolazione dell'art. 311 c.p.p. si è innestata in un contesto nel quale la fase del rinvio «era disciplinata, come quella ordinaria», dall'art. 309 c.p.p., espletando così la sola «funzione» di «regolare la conclusione di detta fase, ossia quella della decisione»: ne deriva che $\mathrm{i}$ «precedenti passaggi procedurali» sono tuttora regolamentati dall'art. 309 c.p.p. ${ }^{98}$. Insomma, il giudizio di rinvio è condotto sulla base dei medesimi «criteri valutativi propri del giudizio ordinario, che presuppongono un pieno esame del materiale probatorio disponibile al momento in cui il giudizio si svolge»; e

96 Cass., Sez. un., 16 luglio 2020, n. 27104, Calella. Processo penale e giustizia, p. 426 ss., 2021. Per un commento, con taluni accenti critici, v. NOCERINO, Wanda. Il dies a quo per la pronuncia del giudizio di rinvio cautelare. Processo penale e giustizia, p. 434 ss., 2021.

97 Cass., Sez. un., 16 luglio 2020, n. 27104, Calella. Processo penale e giustizia, p. 430, 2021, punto 3 del Considerato in diritto.

98 Cass., Sez. un., 16 luglio 2020, n. 27104, Calella. Processo penale e giustizia, p. 430 s., 2021, punto 4 del Considerato in diritto, al quale sono riferite le citazioni nel testo. 
la «sequenza procedurale» si snoda lungo le identiche linee direttrici, tra le quali è compresa la richiesta all'autorità procedente affinché trasmetta nuovamente gli atti che a suo tempo avevano sostenuto la richiesta di misura cautelare e gli eventuali elementi favorevoli sopravvenuti (art. 309 comma 5 c.p.p.). Solo questo incombente, infatti, assicura al giudice di rinvio la disponibilità di tutto il materiale utile per la decisione: non si può dire lo stesso per il fascicolo inviato dalla Corte di cassazione insieme alla sentenza rescindente, perché gli atti ivi contenuti coincidono con quelli trasmessi per la decisione del ricorso ai sensi dell'art. 100 disp. att. c.p.p. e quindi, considerati i limiti propri del giudizio di legittimità, non sono sufficienti ai fini della più ampia valutazione insita nel giudizio di rinvio; inoltre, il riferimento a tali atti ai fini della decisione e della decorrenza del termine per la relativa pronuncia impedirebbe all'autorità procedente di trasmettere al giudice di rinvio gli elementi sopravvenuti durante lo svolgimento del giudizio di cassazione ${ }^{99}$.

Le Sezioni unite - rispondendo a un dubbio espresso nell'ordinanza di rimessione $\mathrm{e}^{100}$ - hanno ulteriormente chiarito che l'applicabilità nel giudizio di rinvio dell'art. 309 comma 5 c.p.p. comporta altresì la sanzione di inefficacia della misura cautelare nel caso di inosservanza del termine indicato dal disposto appena richiamato ${ }^{101}$.

Infine, a parere delle Sezioni unite, la soluzione accolta non è infirmata dalla rilevanza delle esigenze di celerità del procedimento cautelare: queste ultime vanno, infatti, bilanciate con l'esigenza della «completezza degli elementi valutabili per il giudizio, anch'essa rilevante in materia cautelare ed assicurata dall'aggiornamento della disponibilità degli atti, per effetto della trasmissione degli stessi da parte dell'autorità procedente» ${ }^{102}$.

99 Cass., Sez. un., 16 luglio 2020, n. 27104, Calella. Processo penale e giustizia, p. 431,2021 , punto 5 del Considerato in diritto, cui sono collegate le citazioni nel testo.

${ }^{100}$ Cfr. Cass., sez. VI, ord. 16 gennaio 2020, n. 4125, Calella. Sistema penale, 15 luglio 2020, punto 5.2 del Considerato in diritto.

${ }^{101}$ Cass., Sez. un., 16 luglio 2020, n. 27104, Calella. Processo penale e giustizia, p. 432, 2021, punto 5 del Considerato in diritto.

${ }^{102}$ Cfr. ancora Cass., Sez. un., 16 luglio 2020, n. 27104, Calella. Processo penale e giustizia, p. 431, 2021, punto 5 del Considerato in diritto. 
Il parallelismo istituito tra giudizio ordinario di riesame e giudizio di rinvio si riflette sugli atti introduttivi, cioè rispettivamente la richiesta di riesame per la procedura ordinaria e gli atti inviati dalla Corte di cassazione per quella di rinvio: in entrambi i casi, infatti, la ricezione produce come effetto immediato l'inizio della decorrenza del termine di cinque giorni assegnato all'autorità procedente per la trasmissione degli atti su richiesta del presidente del tribunale del riesame. Come per la procedura ordinaria la decorrenza inizia dalla presentazione della richiesta di riesame, così per il giudizio di rinvio la decorrenza inizia quando gli atti trasmessi dalla Corte di cassazione pervengono alla cancelleria centrale del tribunale, non rilevando «il tempo impiegato per il successivo passaggio del fascicolo alla cancelleria della sezione del riesame», costituente «uno dei passaggi burocratici interni all'ufficio giudiziario i cui tempi di espletamento non possono prolungare» la sequenza del procedimento cautelare, che deve essere caratterizzata da «cadenze segnate da tempi certi, che consentano di giungere ad una sollecita definizione» ${ }^{103}$.

In questa pronuncia, le Sezioni unite hanno privilegiato la più compiuta realizzazione del diritto di difesa dell'interessato rispetto all'esigenza di celerità del giudizio di rinvio. Non si può negare, infatti, che, agganciando il dies a quo del termine perentorio stabilito per la decisione alla ricezione degli atti nuovamente richiesti all'autorità procedente, invece che alla ricezione degli atti trasmessi dalla Corte di cassazione, «si provoca inevitabilmente un allungamento del tempo della detenzione della persona sottoposta a misura cautelare» ${ }^{104}$ : va, tuttavia, considerato che, da un canto, l'inosservanza del termine perentorio per l'invio degli atti da parte dell'autorità procedente produce l'inefficacia della misura cautelare; e, dall'altro, la trasmissione degli atti “originari” si accompagna a quella degli elementi favorevoli eventualmente sopravvenuti. Quand'anche si volesse aggiornare l'interpretazione dell'art. 100 disp. att. c.p.p. proprio alla luce dell'art. 311 comma 5-bis c.p.p., sostenendo che si possa oggi giustificare «un invio in Cassazione, quantomeno per i casi che rientrano nello spettro

103 Cass., Sez. un., 16 luglio 2020, n. 27104, Calella. Processo penale e giustizia, p. 432 s., 2021, punto 7 del Considerato in diritto.

${ }^{104}$ In questi termini, criticamente, ALONZI, Fabio. L'importanza della celerità per l'assunzione delle decisioni de libertate. Sistema penale, 15 luglio 2020. 
applicativo» di quest'ultimo disposto, «dell'intero fascicolo della cautela per garantire che, in ipotesi di annullamento, il giudice del rinvio possa adottare tempestivamente la propria decisione seguendo le nuove scansioni temporali» ${ }^{105}$, rimarrebbe sempre aperta la questione delle modalità attraverso cui porre a disposizione di tale giudice gli elementi sopravvenuti favorevoli all'interessato. Secondo l'orientamento minoritario - non avallato dalle Sezioni unite - le «sopravvenienze conoscitive» potrebbero venire depositate in sede di udienza ai sensi dell'art. 309 comma 9 periodo primo c.p.p. ${ }^{106}$, ma in tal modo si applicherebbe l'art. 309 c.p.p. al giudizio di rinvio "ad usum delphini".

Va da ultimo evidenziato un altro profilo, non giunto all'esame delle Sezioni unite, che incide, a monte, sulla celerità del procedimento cautelare pervenuto davanti alla Corte di cassazione ${ }^{107}$. Poiché la riforma del 2015 non ha introdotto modifiche su questo piano, una volta che la Suprema Corte abbia annullato con rinvio ${ }^{108}$ si applica la disposizione generale di

${ }^{105}$ V. ancora ALONZI, Fabio. L'importanza della celerità per l'assunzione delle decisioni de libertate. Sistema penale, 15 luglio 2020, per il quale i vari problemi pratici potrebbero venire risolti attraverso l'impiego della «gestione telematica» degli atti (nello stesso senso NOCERINO, Wanda. Il dies a quo per la pronuncia del giudizio di rinvio cautelare. Processo penale e giustizia, $\mathrm{p}$. $445,2021)$. Una simile modalità, d'altra parte, è ammessa dalla giurisprudenza della Corte di cassazione quanto alla trasmissione degli atti al tribunale del riesame ad opera dell'autorità procedente: v. GIULIANI, Livia. Art. 309. In: ILLUMINATI, Giulio; GIULIANI, Livia (diretto da). Commentario breve al codice di procedura penale. III ed. Milano: Wolters Kluver Cedam, 2020, p. 1423; GIULIANI, Livia. Art. 309. In: ILLUMINATI, Giulio; GIULIANI, Livia (diretto da). Commentario breve al codice di procedura penale. Complemento giurisprudenziale. Appendice di aggiornamento. Milano: Wolters Kluver Cedam, 2020, p. 186, con particolare riguardo alla trasmissione di copia degli atti mediante la posta elettronica certificata.

${ }^{106}$ Cass., sez. I, 29 gennaio 2018, n. 23707, Battaglia.

107 Per la durata media di tale procedimento v. supra, § 3, nota 65.

${ }^{108} \mathrm{Nel} 2019$ sono stati definiti mediante annullamento con rinvio 630 ricorsi in materia di misure cautelari personali, pari al 15\% del totale (4.188). Quanto agli altri esiti, si sono avuti 134 annullamenti senza rinvio (3,2\%), 2.351 inammissibilità $(56,1 \%), 1.042$ rigetti $(24,9 \%)$ e 31 altre decisioni $(0,7 \%)$ : v. Corte Suprema di Cassazione, Ufficio di statistica, La Cassazione penale Annuario statistico 2019, Tab. 4.7 dettaglio uno e Tab. 4.7 dettaglio due. Nel 2020 sono stati definiti mediante annullamento con rinvio 513 ricorsi riguardanti le misure cautelari personali, pari al 13,7\% del totale (3.738). Circa gli 
cui all'art. 625 comma 1 c.p.p. e pertanto la cancelleria «trasmette senza ritardo gli atti del processo con la copia della sentenza al giudice che deve procedere al nuovo giudizio»: ovviamente, la locuzione «senza ritardo» «non rientra nella categoria dei termini e, se la trasmissione non fosse tempestiva, i "tempi morti” si ribalterebbero sul dies a quo da cui inizia a decorrere il termine perentorio di dieci giorni dalla ricezione degli atti, assegnato al giudice di rinvio per la decisione» ${ }^{109}$.

\section{Conclusioni}

Nel riannodare le fila del discorso, per sciogliere il quesito posto nella Premessa, bisogna concludere che neppure le Sezioni unite hanno evitato le oscillazioni da cui sono permeati gli orientamenti delle sezioni semplici, a dimostrazione - se ce ne fosse stato bisogno - che non è facile orientarsi nel bilanciare le esigenze di celerità del giudizio di impugnazione cautelare e la tutela del diritto di difesa dell'indagato/imputato. Si deve, d'altra parte, riconoscere come le tesi giurisprudenziali contrapposte si basino su argomenti solidi, rendendo dunque insoddisfacente, a seconda del punto di vista che si intende privilegiare, l'opzione poi adottata dal Supremo consesso.

Certo, quanto alle pronunce delle Sezioni unite Ramondo e Calella, può osservarsi che i profili da vagliare promanavano da un dato

altri esiti, si sono registrati 93 annullamenti senza rinvio (2,5\%), 2.186 inammissibilità (58,5\%), 917 rigetti (24,5\%) e 29 altre decisioni $(0,8 \%)$ : v. Corte Suprema di Cassazione, Ufficio di statistica, La Cassazione penale - Annuario statistico 2020, Tab. 5.6 dettaglio uno e Tab. 5.6 dettaglio due.

${ }^{109}$ Cfr. BARGIS, Marta. Le novità nella disciplina del giudizio di rinvio: una replica imperfetta. Disponibile in: http://www.lalegislazionepenale.eu, 22 settembre 2015, p. 9 (v. pure p. 14-16). In senso adesivo MAGGIO, Paola. Le impugnazioni delle misure cautelari personali. Milano: Giuffrè Francis Lefebvre, 2018, p. 497 s.; MARZADURI, Enrico. Diritto di difesa e tempi del procedimento dinanzi al tribunale della libertà. In: GIULIANI, Livia (a cura di). La riforma delle misure cautelari personali. Torino: Giappichelli, 2015, p. 246, nota 124. Affrontano il problema, più di recente, COLAIACOVO, Guido. I tempi del giudizio di rinvio in materia cautelare (a margine di una recente sentenza della Suprema Corte). Sistema penale, 21 gennaio 2020; NOCERINO, Wanda. Il dies a quo per la pronuncia del giudizio di rinvio cautelare. Processo penale e giustizia, p. 446, 2021. 
letterale non felice introdotto dalla riforma del 2015, che ha reso perciò inevitabile il tentativo di coniugarlo con il contesto sistematico. Se gli approdi rimangono opinabili, solo il legislatore potrebbe porre mano alle dovute precisazioni, fornendo in altre parole una sorta di interpretazione autentica - poco importa che avalli oppure no il dictum delle Sezioni unite - al fine di sopire le questioni interpretative controverse.

Ma il discorso non muta per la pronuncia delle Sezioni unite Salvati riguardante la misura cautelare disposta in sede di appello e la necessità o no dell'interrogatorio di garanzia: pure qui un intervento legislativo - del resto variamente auspicato in dottrina ${ }^{110}$ - si rivelerebbe oltremodo opportuno.

\section{Bibliografia}

ALONZI, Fabio. Un richiamo al rigore nell'interpretazione delle norme de libertate. Processo penale e giustizia, p. 259-268, 2018.

ALONZI, Fabio. L'importanza della celerità per l'assunzione delle decisioni de libertate. Sistema penale, 15 luglio 2020.

ALVINO, Francesco. Incompetenza per territorio, urgenza cautelare e interesse del pubblico ministero alle relative contestazioni nelle impugnazioni de libertate. Sistema penale, n. 9, p. 125-143, 2020.

ANGIOLINI, Giulia. Il "nuovo" art. 309 comma 10 c.p.p. supera l'esame di costituzionalità (ma non ancora quello della prassi applicativa). Diritto penale contemporaneo, 6 dicembre 2016.

ARASI, Simona. L'interrogatorio di garanzia in caso di applicazione di misura cautelare coercitiva da parte del Tribunale del riesame in accoglimento dell'appello del Pubblico Ministero: obbligo o facoltà? Processo penale e giustizia, p. 84-93, 2021.

BARGIS, Marta. L'appello cautelare: un innesto per rifrazione. Disponibile in: http://www.lalegislazionepenale.eu, 22 settembre 2015, p. 1-7.

BARGIS, Marta. Le novità nella disciplina del giudizio di rinvio: una replica imperfetta. Disponibile in: http://www.lalegislazionepenale.eu, 22 settembre 2015, p. 1-16.

${ }^{110}$ Cfr. supra, $\S 3$, nel testo e note 84 e 87. 
BLEFARI, Chiara Rosa. Le Sezioni Unite si pronunciano sull'interesse a ricorrere del Pubblico ministero, nel caso in cui il Giudice del riesame, rilevata l'incompetenza del Giudice che ha emesso l'ordinanza, la annulli. Processo penale e giustizia, p. 106-113, 2021.

CARDAMONE, Daniela. Il sistema dei rimedi de libertate alla luce della giurisprudenza della Cedu nella sentenza Rizzotto c. Italia (5 settembre 2019). Questione giustizia, $1^{\circ}$ ottobre 2019.

CASSIBBA, Fabio. Impugnazioni de libertate e garanzie minime dell'equità processuale. Rivista italiana di diritto e procedura penale, p. 968-971, 2018.

COLAIACOVO, Guido. I tempi del giudizio di rinvio in materia cautelare (a margine di una recente sentenza della Suprema Corte). Sistema penale, 21 gennaio 2020.

COLAIACOVO, Guido. Latitanza e riesame (appunti a margine di una sentenza della Corte europea dei diritti dell'uomo). Cassazione penale, p. 768-772, 2020.

CURZIO, Piero. Relazione sull'amministrazione della giustizia nell'anno 2020. Roma, 29 gennaio 2021, p. 73.

DE ROBBIO, Costantino. Applicazione di misura cautelare da parte del tribunale del riesame e interrogatorio di garanzia: la sentenza delle Sezioni unite. Sistema penale, 15 giugno 2020.

DEL COCO, Rosita. Art. 618. In: ILLUMINATI, Giulio; GIULIANI, Livia (diretto da). Commentario breve al codice di procedura penale. III ed. Milano: Wolters Kluver Cedam, 2020, p. 3050 s.

DI DOMENICO, Alessia. Le Sezioni Unite sul deposito del ricorso in sede cautelare: un'occasione per chiarire la corretta interpretazione dell'art. 311 c.p.p. Sistema penale, 4 febbraio 2021.

GATTO, Antonio. Il diritto dell'imputato detenuto di presenziare all'udienza di riesame al vaglio delle Sezioni unite. Sistema penale, n. 2, p. 17-45, 2020.

GERARDI, Alessandro. Il luogo delle impugnazioni cautelari di legittimità: la stretta delle Sezioni unite. Archivio penale (web), n. 1, p. 1-23, 2021.

GIULIANI, Livia. Autodifesa e difesa tecnica nei procedimenti de libertate. Padova: Cedam, 2012, p. 190-198.

GIULIANI, Livia. Art. 309. In: ILLUMINATI, Giulio; GIULIANI, Livia (diretto da). Commentario breve al codice di procedura penale. III ed. Milano: Wolters Kluver Cedam, 2020, p. 1423 e 1433 ss. 
GIULIANI, Livia. Art. 309. In: ILLUMINATI, Giulio; GIULIANI, Livia (diretto da). Commentario breve al codice di procedura penale. Complemento giurisprudenziale. Appendice di aggiornamento. Milano: Wolters Kluver Cedam, 2020, p. 186.

GREVI, Vittorio; CERESA-GASTALDO, Massimo. Misure cautelari. In: BARGIS, Marta (a cura di). Compendio di procedura penale, X ed. Milano: Wolters Kluver Cedam, 2020, p. 372.

GRISONICH, Elisa. Il diritto dell'imputato detenuto a partecipare personalmente all'udienza di riesame: le Sezioni Unite delineano le modalità di esercizio della garanzia dopo la 1. n. 47 del 2015. Sistema penale, 24 aprile 2020.

ILLUMINATI, Giulio. Verso il ripristino della cultura delle garanzie in tema di libertà personale dell'imputato. Rivista italiana di diritto e procedura penale, $\mathrm{p}$. 1156-1162, 2015.

MAGGIO, Paola. Le impugnazioni delle misure cautelari personali. Milano: Giuffrè Francis Lefebvre, 2018.

MARANDOLA, Antonella. Partecipazione personale al riesame: una lettura in linea con la Costituzione. Giurisprudenza italiana, p. 1932 ss., 2019.

MARANDOLA, Antonella. Misura cautelare applicata dal tribunale della libertà in sede d'appello: negato l'interrogatorio di garanzia. Cassazione penale, p. 40364040, 2020.

MARZADURI, Enrico. Diritto di difesa e tempi del procedimento dinanzi al tribunale della libertà. In: GIULIANI, Livia (a cura di). La riforma delle misure cautelari personali. Torino: Giappichelli, 2015, p. 211-248.

MITTICA, Francesca Romana. La partecipazione all'udienza di riesame dopo la 1. n. 47 del 2015. Processo penale e giustizia, n. 3, p. 94-106, 2016.

NASCIMBENE, Bruno (a cura di). Unione europea. Trattati. Torino: Giappichelli, 2010, p. 381 s.

NOCERINO, Wanda. Il dies a quo per la pronuncia del giudizio di rinvio cautelare. Processo penale e giustizia, p. 434-446, 2021.

PAULESU, Pier Paolo. Vittima del reato e processo penale: uno sguardo d'insieme (informazioni, diritti, tutele). In: BARGIS, Marta; BELLUTA, Hervé (a cura di), Vittime di reato e sistema penale. Torino: Giappichelli, 2017, p. 158.

PERONI, Francesco. Davvero superfluo l'interrogatorio di garanzia, in caso di cautela adottata in sede d'appello?. Diritto penale e processo, p. 60-67, 2021. 
PRESSACCO, Luca. Latitanza e "giusto processo cautelare". Rivista italiana di diritto e procedura penale, p. 363-367, 2020.

RAFARACI, Tommaso. Le garanzie del procedimento de libertate: il diritto ad ottenere in un termine congruo le decisioni sulla libertà personale. In: BALSAMO, Antonio; KOSTORIS, Roberto E. (a cura di). Giurisprudenza europea e processo penale italiano. Torino: Giappichelli, 2008, p. 271-291.

ROMANELLI, Bartolomeo. Il differimento dell'udienza di riesame tra esigenze di difesa sostanziale e limiti all'impugnazione. Diritto penale contemporaneo, 8 giugno 2016, p.1-10.

SPANGHER, Giorgio. Un restyling per le misure cautelari. Diritto penale e processo, p. 531-534, 2015.

SPANGHER, Giorgio. Brevi riflessioni sistematiche sulle misure cautelari dopo la 1. n. 47 del 2015. Diritto penale contemporaneo, Rivista trimestrale, n. 4, p. 6, 2015.

TARALLO, Antonio. "Unicità dell'impugnazione", "giudicato cautelare" e tutela dei latitanti: qualche perplessità sulle scelte della Corte e.d.u. Processo penale e giustizia, p. 418 ss., 2020.

TECCE, Raffaele, Il diritto del detenuto a partecipare all'udienza di riesame. Processo penale e giustizia, p. 1412-1421, 2020.

URBINATI, Francesco. Interrogatorio di garanzia e misure cautelari: configurazioni processuali. Archivio penale (web), n. 2, p. 1-22, 2020.

VARONE, Fabio. A proposito della fungibilità delle garanzie: le Sezioni unite negano il diritto dell'accusato all'interrogatorio in caso di applicazione delle misure coercitive a seguito di appello del pubblico ministero. Archivio penale (web), n. 2, p. 1-18, 2020.

ZACCHÈ, Francesco. Le cautele fra prerogative dell'imputato e tutela della vittima di reati violenti. Rivista italiana di diritto e procedura penale, p. 675 ss., 2015. 


\section{Additional information and author's declarations (scientific integrity)}

Conflict of interest declaration: the author confirms that there are no conflicts of interest in conducting this research and writing this article.

Declaration of authorship: all and only researchers who comply the authorship requirements of this article are listed as authors; all coauthors are fully responsible for this work in its entirety.

Declaration of originality: the author assures that the text here published has not been previously published in any other resource and that future republication will only take place with the express indication of the reference of this original publication; she also attests that there is no third party plagiarism or self-plagiarism.

\section{Editorial process dates}

(http://www.ibraspp.com.br/revista/index.php/RBDPP/about/editorialPolicies)

- Submission: $13 / 07 / 2021$

- Desk review and plagiarism check: 02/08/2021

- Final editorial decision: 04/08/2021

- Final version: 04/10/2021
Editorial team

- Editor-in-chief: 1 (VGV)

- Associated-editor: 2 (BC, LG)

- Autrice invitata

\section{HOW TO CITE (ABNT BRAZIL):}

BARGIS, Marta. Le impugnazioni cautelari nel recente "attivismo" delle Sezioni unite della Corte di cassazione. Revista Brasileira de Direito Processual Penal, vol. 7, n. 3, p. 1935-1976, set./dez. 2021. https://doi.org/10.22197/rbdpp.v7i3.629



Esta obra está licenciada com uma Licença Creative Commons Atribuição-NãoComercial 4.0 Internacional. 